\title{
»Agrarwende«: Über den institutionellen Umgang mit den Folgeproblemen der Folgenreflexion im Agrarbereich
}

\author{
Von Cordula Kropp und Jost Wagner
}

Zusammenfassung: Rinderwahnsinn (BSE) und Maul- und Klauenseuche (MKS), die großen Risikoskandale der Jahre 2000/01, haben im Agrarbereich zu einer massiven Erschütterung und Infragestellung industriemoderner Routinen und Selbstverständnisse geführt. In der Folge geriet der gesamte Bereich unter erheblichen Veränderungs- und Legitimationsdruck, den die rot-grüne Regierungspolitik als »window of opportunity« nutzte, um einen politischen Paradigmenwechsel, die »Agrarwende«, anzukündigen. Diese »tief greifende Neuorientierung « wurde vom Kanzler, seiner Ministerin und dem Mediendiskurs als mit der Krise fällig gewordene Öffnung eines bis dato abgeschirmten Bereiches für die Notwendigkeiten (ökologischer) Modernisierung dargestellt. Ausgehend von dem in der Theorie reflexiver Modernisierung ausbuchstabierten Nebenfolgentheorem geht es in dem Beitrag demgegenüber darum, die »Agrarwende « in den Kontext eines für Risikogesellschaften typischen Meta-Wandels elementarer Bezugsrahmen zu setzen und das Verhältnis von intendierter Transformation und reflexhafter Anpassung an die Nebenfolgeproblematik zu diskutieren. Dazu werden exemplarisch die institutionell weitreichenden Herausforderungen von Folgenreflexion und daran orientiertem Umbau benannt sowie die Erfolgschancen und institutionellen Hemmnisse der politisch initiierten »Agrarwende« im Bereich von Agrarwissenschaft und -politik diskutiert. Inwieweit können, so die zentrale Fragestellung, in den Agrarwissenschaften, in der agrarpolitischen Entscheidungsfindung und im Dialog dieser beiden miteinander neue grundsätzliche Rahmungen der Orientierung an Nichtwissen, Bewertungsambivalenz und Folgensensibilität erfolgreich verankert werden? Indem die eigendynamischen Prozesse der Verarbeitung der Erosion bisheriger Selbstverständnisse und Basisüberzeugungen sowie der Umund Übersetzung der neuen gesellschaftlichen Ansprüche in den agrarwissenschaftlichen und agrarpolitischen Institutionen herausgearbeitet werden, wird ein Verständnis der beobachtbaren Phänomene von »Integration « und »Ableitung « durch die Verknüpfung von institutionentheoretischen Konzepten mit der Theorie Reflexiver Modernisierung angestrebt.

Skandale und Risiken im Agrarsektor sind nicht neu. Aber erst die BSE-Krise und die erschreckenden Bilder der gewaltigen, apokalyptisch anmutenden Scheiterhaufen, auf denen im Sommer 2001 Abertausende von Rindern - nach Vorgabe der EU-Agrarpolitik - verbrannt wurden, weil sie durch die grassierende Maul- und Klauenseuche (MKS) bedroht waren, haben Zweifel und Misstrauen in Agrarwissenschaft und -politik in einer bis dahin nicht gekannten Intensität hervorgebracht. Dabei kam das Auftreten der Maul- und Klauenseuche aber nicht überraschend und ohne Vorwarnung, sondern erscheint in besonderer Weise als zivilisatorisch hergestellte und dennoch als »natürlich « ausgegrenzte und unverantwortete Nebenfolge (vgl. Latour 2004).

MKS wurde zusammen mit BSE, Nitrofen und vielen kleineren Agrarskandalen der letzten Jahre zum Kristallisationspunkt der Kritik an einem Bereich, der bis dahin zumindest in seinem Zentrum stark von der Logik eines industriemodernen »Weiter-So« geprägt war. Zumutungen der Umorientierung - etwa unter dem Druck des Leitbilds Nachhaltiger Entwicklung - konnten dort bislang weitgehend zurückgewiesen werden. Durch die großen öffentlichen Skandale, ihren hohen Takt und die Art ihrer diskursiven Rahmung wurden nun aber die Nebenfolgen der Erfolge bisheriger landwirtschaftlicher Entwicklung unter den Zeichen von globaler Wettbewerbsfähigkeit und technisch-industriellem Fortschritt deutlicher denn je und stellten die bisherigen Handlungsorientierungen und ihre zentralen Institutionen massiv in Frage. 
In der Konsequenz geriet der gesamte Bereich unter erheblichen Veränderungs- und Legitimationsdruck, den die rot-grüne Regierungspolitik als »window of opportunity« nutzte, um einen politischen Paradigmenwechsel, die »Agrarwende«, anzukündigen. Diese »tief greifende Neuorientierung « wurde vom Kanzler, seiner Ministerin und dem Mediendiskurs im Rahmen der gewählten Wende-Rhetorik als Abkehr von »industriellen Produktionsweisen« in der Landwirtschaft angekündigt. Tatsächlich rief der Bundeskanzler Gerhard Schröder nur sechs Tage, nachdem am 24. November 2000 eine BSE-Erkrankung bei einem in Deutschland geborenen Rind festgestellt wurde, dazu auf, die Krise zu nutzen, »um eine Perspektive für eine andere, verbraucherfreundliche Landwirtschaft $\mathrm{zu}$ entwickeln, also weg von den Agrarfabriken zu kommen« (zit. nach Feindt/Ratschow 2003: 5). Peter Feindt und Christiane Ratschow kommen in ihrer politikwissenschaftlichen Analyse von Agrarwendediskurs und institutionellem Politikwechsel allerdings zu der Einschätzung, aufgrund der institutionalisierten Entscheidungsarenen und der bestehenden Verhandlungssysteme ${ }^{1}$ seien einer »relativ radikalen, modernisierungskritischen Diagnose« nur eher inkrementalistisch anmutende, »kleine Schritte« gefolgt (ebd. 10). Ähnlich resümiert auch Peter Oosterveer (2002) in seiner vergleichenden Studie der politischen Reaktionen auf die BSE-Krise in Großbritannien, den Niederlanden, Frankreich und Deutschland: »it goes one bridge too far to conclude from the BSE story that new reflexive risk politics are there« (ebd.: 227).

Ausgehend von dem in der Theorie reflexiver Modernisierung ausbuchstabierten Nebenfolgentheorem geht es in unserem Beitrag demgegenüber darum, die »Agrarwende« und ihr institutionelles Schicksal weder allein aus politischen Gelegenheitsstrukturen oder organisatorischen und personalen Restriktionen zu erklären, noch im Hinblick auf neue Formen der staatlich organisierten Politik zu betrachten, sondern in den Kontext eines für Risikogesellschaften typischen Meta-Wandels elementarer Bezugsrahmen zu setzen. ${ }^{2}$ So interessiert uns insbesondere das Verhältnis von politisch intendierter Veränderung im Sinne einer weit reichenden Reform (»Agrarwende« als policy change) zu reflexhafter, schleichender und oft ungewollter Transformation als eigendynamische Konsequenz aus Nebenfolgen und Nebenfolgenverarbeitung. Die sozialwissenschaftliche Literatur zitiert die BSE-Krise gerne als Paradebeispiel der Risikogesellschaft. Sie wird als ein Resultat zivilisatorisch hergestellter Ungewissheit im Rahmen von landwirtschaftlicher Intensivproduktion, technischer Entwicklung der Futtermittelindustrie und globalen Handelsverflechtungen beschrieben, deren vielfältige Wahrnehmung sich nicht nur an unterschiedlichen Interessen betroffener Akteursgruppen bricht, sondern auch an der politischen Leugnung sowie an höchst umstrittenen Bewertungen einer überforderten Wissenschaft (vgl. Hinchliffe 2001, Viehöver 2000). Lässt sich aber auch ihre Verarbeitung in den Institutionen der Öffentlichkeit, der Politik und der Wissenschaft als reflexiv-moderner Meta-Wandel beschreiben, der weder in einfachen Nachbesserungs- und Anpassungsbemühungen aufgeht, noch in die befürchtete frühagrarische Gegenmoderne führt (vgl. auch Mol/Bulkeley 2002)? Welche interpretativen Vorteile bringt eine solche Betrachtung gegenüber dem politisch reklamierten Anspruch, in Reaktion auf eine Krise die politische Gelegenheitsstruktur für eine Korrektur der agrarpolitischen Leitvorstellungen und der landwirtschaftlichen Gepflogenheiten genutzt zu haben?

Nach dem Aufdecken des ersten >hausgemachten< deutschen BSE-Falles gab die neue Verbraucherschutzministerin Renate Künast im Februar 2001 eine Regierungserklärung ab, die sie mit dem Satz einleitete: »Der BSE-Skandal markiert das Ende der Landwirtschaftspo-

1) Mehrebenenpolitik mit einer in diesem Politikfeld einzigartigen Verflechtung von EU-, Bundes- und Länderpolitik (vgl. Gerlach et al. 2005)

2) Diese Diskussion basiert auf dem durch das BMBF geförderten Forschungsprojekt »Wissen für Entscheidungsprozesse - Ansätze für eine dialogisch-reflexive Schnittstellenkommunikation zwischen Wissenschaft und Politik« (4/2004-3/2007). 
litik alten Typs. Wir stehen [...] vor einem Scherbenhaufen $\ll .{ }^{3}$ Sehr deutlich markierte sie im Anschluss einen Neubeginn: die Agrarwende, die nach dem Scheitern der bisherigen Politik eine »Agrarpolitik neuen Typs« eröffnen solle. Renate Künast folgt damit, wie die meisten Politiker und auch manche Politikwissenschaftler, einer politikzentrierten Betrachtung, in der die Politik als Subjekt sowohl für die Verursachung von Krisen als auch für deren Wiedergutmachung und Behebung zentral verantwortlich ist - und nicht bspw. die Landwirtschaft oder die Futtermittelindustrie. Diese Sichtweise haben im Fall der deutschen BSEKrise auch die Medien mit ihrem Bedürfnis nach Skandal, Bösewicht und Retterfigur übernommen (vgl. Kleinschmidt/Feindt 2004). Skeptischer gegenüber diesem Steuerungs- und Neuerungsanspruch und zugleich »optimistischer in Hinblick auf Reichweite und Kontinuität eines subjektlosen Wandels fragen wir aus der Perspektive der Theorie Reflexiver Modernisierung, inwieweit hinter dem Rücken auch der selbsternannten Kommandozentralen in Politik, Wissenschaft und Öffentlichkeit die BSE- und MKS-Krisen einer Transformationsdynamik neuen Schwung gaben und geben, die bereits durch viele vorhergehende Risiken und Nebenfolgen in der modernisierten Landwirtschaft angetrieben wurde. Schließlich hat die durch diese Dynamik hervorgerufene sukzessive Zersetzung und Pluralisierung von agrarpolitischen Leitideen und ideellen Grundkategorien der späteren »Agrarwende« das kognitive und institutionelle Material an Alternativen geliefert. So betrachten wir die »Agrarwende « nicht nur als politische Reaktion auf eine politische Krise mit diskutablen Konsequenzen in Landwirtschaft, Agrarwissenschaft und Agraröffentlichkeit, sondern als teilweise integriertes, teilweise abgelehntes, teilweise gleichsinnig und teilweise quer liegendes Wende-Motiv, dessen Chancen und Grenzen erst unter Sichtung des tiefer liegenden Meta-Wandels der Moderne auch im Agrarbereich verständlich werden. Umgekehrt hoffen wir, auch die Theorie der reflexiven Moderne durch eine Betrachtung bereichern zu können, die intendierte Reformen bzw. gezielte »Risikopolitik« mit dem schleichenden Meta-Wandel der Bezugsrahmen im Zuge reflexiver Modernisierung verknüpft und so erlaubt, erste, zunächst fallspezifische Hypothesen über das »Sowohl-als-Auch« neuer und alter Formen des institutionellen Problemlösens aufzustellen.

\section{Folgeprobleme der Folgenprobleme aus Sicht der Theorie Reflexiver Modernisierung}

Folgt man der Theorie Reflexiver Modernisierung, handelt es sich bei den BSE- und MKSKrisen und der durch sie angestoßenen »Agrarwende « weniger um einen alleinstehenden Wahrnehmungs- und Politikwechsel aufgrund böser Überraschungen und bestimmter parteipolitischer Konstellationen als um den Ausdruck eines auch in anderen Bereichen allenthalben anzutreffenden Prozesses der radikalisierten und entgrenzten »Modernisierung der Moderne $\ll(B e c k / B o n ß 2001)$.

»Modernisierung der Moderne« bedeutet zunächst ganz allgemein, dass im Zuge fortgeschrittener Modernisierung die vor allem westlich-industriell geprägte Erste Moderne und ihre Leitvorstellungen (von wissenschaftlich-technischem Fortschritt, von Kontrolle und Sicherheit, von nationalen Wohlfahrtsdemokratien und arbeitsmarktzentrierter Selbstverwirklichung), ihre vermeintlich endgültigen Grundkategorien (bspw. der eindeutigen Zuordnung und der wissenschaftlich definierten Rationalität) sowie ihre als fraglos und notwendig erachteten, oft essentialistisch begründeten Schlüsselinstitutionen nun mit den eigenen Folgen und nicht-intendierten Nebenfolgen konfrontiert werden. Damit wendet sich die Modernisierung, erst latent und dann immer manifester, erzwungenermaßen »reflexiv« nach innen und wird sich »selbst zum Thema und Problem«, so die Kernaussage der seit zumindest

3) Regierungserklärung zur neuen Verbraucherschutz- und Landwirtschaftspolitik, 8. Februar 2001. 
1986 von Ulrich Beck und in seinem Umkreis weiter entwickelten Gesellschaftsdiagnose (vgl. insb. Beck 1986, 1993, Beck/Bonß 2001, Beck/Lau 2004). Dabei werden Selbstanwendung und Selbstkonfrontation im Rahmen einer verselbständigten Modernisierungsdynamik ${ }^{4}$ (das »Theorem der Eigendynamik«; vgl. Beck/Grande 2004: 52) von nicht gewollten, nicht gesehenen und nicht begrenzbaren Nebenfolgen und Risiken, wie etwa BSE, begleitet, unterstützt und vorangetrieben (das Nebenfolgentheorem, ebd.).

Solcherlei Nebenfolgen und zivilisatorisch verursachte Risiken sprengen in ihrer Wirkung nicht nur zeitlich, sozial und räumlich die institutionell gesetzten Grenzen, sondern wirken als Nebenfolgen der Nebenfolgen auch über Funktionssysteme hinaus zersetzend und politisierend. Indem insbesondere Risiken und ihre strittige Wahrnehmung die Problemlösungskompetenz der etablierten Institutionen vor Herausforderungen stellen, die in den bestehenden Formaten nicht gelöst werden können, entkernen sie Handlungssicherheiten und delegitimieren die institutionalisierten Hüter von Wohlstand und Sicherheit, insbesondere in Wissenschaft und Politik. Nicht, wie in der Ersten Moderne erwartet, ein »increase of mastery « so Bruno Latour (2003: 38), »but an heightened awareness that mastery is impossible«, ist das vorläufige Ende der Geschichte. Auf diese Weise münden die durch Modernisierung entfesselten und verselbständigten Eigendynamiken, bspw. des globalen Wettbewerbs oder der rasanten wissenschaftlich-technischen Veränderung unserer Welt, zusammen mit den in ihnen hergestellten Ungewissheiten und Ambivalenzen zunächst in die Risikogesellschaft. Diese sorgt sich nicht länger um die Verteilung von Reichtum, sondern um die Verteilung von Risiken und führt schließlich in die reflexive Zweite Moderne: In ihr werden nun bisher fraglose Selbstverständlichkeiten, Grundüberzeugungen und Eindeutigkeiten pluralisiert und politisiert.

Im Rahmen einer solchermaßen reflexiven Modernisierung geraten ungeplant und schleichend, d.h. vorbei an den Parlamenten und den steuerungsverliebten Akteursgruppen, gerade jene Grundunterscheidungen und Schlüsselinstitutionen in einen Prozess der Revidierung, Restrukturierung und Neuverhandlung, die Selbstverständnis und »Erfolg« der so genannten Ersten Moderne prägten. Im Agrarbereich sind dies vor allem die stabilisierten, von Verantwortung entlastenden Unterscheidungen von Natur und Gesellschaft sowie von menschlichen und nichtmenschlichen Wesen, aber auch die eindeutige Differenz von primärem und tertiärem Sektor, von Wissenschaft, Landwirtschaft und Politik sowie in jüngerer Zeit von funktionalen Produktionsstätten und traditionalen Höfen. Schließlich gehörten das Versprechen von Wohlstand, Sicherheit und Modernität durch wissenschaftlich-technisch vorangetriebene Produktivitätssteigerung, deren nationale Regulierung, usf dazu. Diese kategorialen, meist dual strukturierten Grundkategorien und Leitideen des Wandels stehen im Übergang in die Zweite Moderne im Agrarbereich wie auch generell nicht mehr fraglos und unangefochten im Raum. Sie sind selbst der Begründungs- und Legitimationspflicht als kontinuierlich fortgeführte Basisprinzipien ${ }^{5}$ unterworfen und werden Gegenstand konfliktreicher Neudefinitionen. In diesem Sinne handelt es sich um einen Meta-Wandel auf der übergeordneten Ebene der institutionellen Bezugsrahmen und deren Fundierung, im Rahmen dessen Institu-

4) Unter dieser Modernisierungsdynamik wird insbesondere das exponentielle Wachstum von Wissenschaft, Technik, Mobilität, individualisierter Subjektivität, entgrenzten Kapitalströmen etc. diskutiert (vgl. Beck/Grande 2004; Beck/Lau 2004 sowie Kratzer in diesem Heft).

5) Daher werden sie aber auch nicht beliebig im Sinne eines postmodernen »anything goes «. Die Theorie reflexiver Modernisierung stellt heraus, dass zwar die Basisinstitutionen erodieren und konfliktuös werden, nicht jedoch die ihnen zugrunde liegenden Basisprinzipien, die die »kognitive Infrastruktur « gleichermaßen der Ersten und Zweiten Moderne bilden (vgl. Beck/Lau in diesem Heft). So müssen neue Unterscheidungen etwa nach wie vor dem Prinzip der Begründungspflicht folgen, können nicht willkürlich gesetzt werden. 
tionen und Organisationen u.U. äußerlich unverändert erscheinen, von innen aber konfliktreichen Prozessen der De- und Re-Strukturierung ausgesetzt sein können.

Nun liefert die Theorie der reflexiven Modernisierung für das Nebenfolgentheorem zweierlei Interpretationen, in denen auch der Prozess des von Risiken und Nebenfolgen verursachten Institutionenwandels unterschiedlich beschrieben wird (vgl. Beck/Bonß/Lau 2001: 32f.). Zum einen wird von einer »Gegenmacht der Gefahr« (Beck 1988) gesprochen, mit der Risiken und hergestellte Ungewissheiten wirtschaftliche und politische Institutionen wie Versicherungen vor Probleme stellen, die sie mit den überkommenen Mitteln nicht lösen können: Nebenfolgen wie BSE oder der Keulungsimperativ im Gefolge von MKS irritieren die Interpretationen, Entscheidungsroutinen und Rationalitätsgrundlagen der bestehenden Organisationen. Sie werden insbesondere von der prinzipiellen Uneindeutigkeit und den weitläufigen Interdependenzen ${ }^{6}$ überfordert. So kommt es zu einer »institutionellen Funktionskrise« (Beck/Bonß/Lau 2001: 33), die »materiale institutionelle Innovation« (ebd.) erfordert und unter günstigen Bedingungen in einen Umbau der Institutionen mündet, wie wir ihn im Fall der Agrarwende beobachten konnten und unten näher beschreiben.

Die zweite Interpretation der Nebenfolgendynamik hebt stärker auf die Wahrnehmungsund Definitionsabhängigkeit von Risiken ab. So ist die institutionelle Verarbeitung dieser soziokulturellen Konstrukte letztlich von langen, stark politisierten Konstruktionsprozessen begleitet (vgl. zu BSE bspw. Viehöver 2000). Je offensichtlicher sich ein Risiko den wissenschaftlichen Methoden und politischen Institutionen der Risiko-Einschätzung und des Risiko-Managements entzieht, desto mehr Einfluss gewinnt die stets plurale Risikowahrnehmung und die stets interessierte Risikoinszenierung. So bestehen Risiko und Skandal von BSE nicht in der Zahl der toten und erkrankten Menschen und Rinder, im objektiven Verlauf seiner Ausdehnung oder im wirtschaftlichen Verlust, sondern sind Ausdruck einer gesellschaftlichen »Anerkennungskarriere « im Gerangel von Politik, Wissenschaft, Fachöffentlichkeit und Betroffenen. Sie sind auch Ausdrucksform immer raumgreifenderer »Interdependenz und forcieren dieselbe. Anders als globale Produktions- und Lebensmittelketten, die latent bleiben können, durchbrechen globale Risiken mit ihrer physisch und politisch entgrenzten Explosivität diese Latenz« (Beck/Grande 2004: 298) - und schaffen unter bestimmten Bedingungen Öffentlichkeiten, die sich dieser Interdependenz bewusst werden (»reflexive Globalität«; ebd.). So kommt es im Rahmen von Risikodiskursen zu »Legitimationskrisen « in den Institutionen (vgl. Beck/Bonß/Lau 2001: 33), die weder durch symbolische Beschwichtigung oder Leugnung noch mit den klassischen Mitteln der wissenschaftlichen Beweisführung eingedämmt werden können. Stattdessen wird die Politisierung der Nebenfolgen, zumindest so lange sie ein Thema der Massenmedien sind, relativ unabhängig von ihrem Gefahrengehalt durch die gegenläufigen Interessen der betroffenen Akteursgruppen sowie die Turbulenzen des öffentlichen Diskurses vorangetrieben (ebd.).

Wir meinen, im von uns untersuchten Fall sind beide Nebenfolgendynamiken, die der institutionellen Funktionskrise im Gewande der »Agrarwende« und die der institutionellen Legitimationskrise unter dem Mantel vielfältiger De- und Re-Strukturierungsprozesse in den betroffenen Institutionen am Werk. Beide Prozesse interagieren, sind sich wechselseitig Nahrung als auch Gift, durchdringen sich, behindern sich, verändern sich. In der Konsequenz zeichnet sich ein »Sowohl-als-Auch« alter, neuer und reflexiv neuer Verfahren der institutionellen Problembehandlung und Entscheidungsbegründung ab, ein kompliziertes Wechselspiel von Nebenfolgen, Nebenfolgenreflexion und Reflexion der Nebenfolgenrefle-

6) Renn/Dreyer/Klinke (2003: 5) stellen heraus, dass gerade »the embeddedness of risks to human health and the environment in a larger context of social, financial and economic risks and opportunities « systemische Risiken wie BSE zur besonderen Herausforderung für die klassischen Institutionen werden lässt. 
xion, zu dessen Verständnis wir anhand unserer Überlegungen zum Agrarbereich beitragen möchten. Dabei ist für uns auch eine entscheidende Frage, wie es den betroffenen Institutionen und Organisationen in Wissenschaft und Politik gelingen kann, sich den komplexen Herausforderungen erfolgreich zu stellen (vgl. Wagner/Kropp 2005).

\section{Die Industrialisierung der Landwirtschaft und ihre Krisen: Erste und Zweite Moderne im Agrarbereich}

Um das Zusammenspiel von politisch formulierter Agrarwende und sich gleichzeitig vollziehendem Meta-Wandel im Agrarbereich zu verstehen, werden wir in diesem Abschnitt die speziellen Hintergründe der Institutionenentwicklung im Agrarbereich in Deutschland herausarbeiten, die sich geradezu exemplarisch vor der Interpretationsfolie einer Ersten Moderne rekonstruieren lassen. Vielfach ist der Übergang von der vormodernen zur modernen Gesellschaft als Ablösung der Agrargesellschaft durch die Industriegesellschaft beschrieben worden. Dabei bot jedoch gerade die massive Steigerung der landwirtschaftlichen Produktion, das damit verbundene Bevölkerungswachstum und die Freisetzung von Arbeitskräften die notwendige Basis der Prozesse, die uns heute als Industrialisierung bekannt sind (Kötter 1997). Mit der Moderne hat die Landwirtschaft zwar ihre zentrale gesellschaftsprägende Stellung verloren, sie blieb jedoch nicht im vormodernen Sektor zurück, sondern verfolgte nun ihrerseits eine Modernisierungsstrategie, die lange Zeit das Selbstverständnis des Agrarbereichs und der zugehörigen wissenschaftlichen und politischen Akteure dominierte und teilweise bis heute prägt. ${ }^{7}$

Diese Modernisierungsstrategie stand von Anfang an in einem engen Zusammenhang mit der Herausbildung einer eigenen wissenschaftlichen Disziplin. Als deren Geburtsstunde gilt die Begründung der Landwirtschaftlichen Betriebslehre durch Albrecht Thaer (1752 - 1828), deren maßgebliches Vorhaben darin bestand, den landwirtschaftlichen Betrieb aus seiner Sonderstellung herauszuheben und mit anderen produzierenden Unternehmen gleichzusetzen »Die Landwirthschaft ist ein Gewerbe, welches zum Zweck hat, durch Production [...] Gewinn zu erzeugen. Je höher dieser Gewinn nachhaltig ist, desto vollständiger wird dieser Zweck erfüllt« (Thaer 1809). Die von Thaer eingeführten Innovationen, wie etwa der Wegfall der Brache durch die Dreifelderwirtschaft und der verstärkte Einsatz von Stallmist als Dünger, dienten denn auch in erster Linie der Produktionssteigerung und Gewinnmaximierung des »Unternehmens Bauernhof « durch standardisierte Methoden. Die Aufgabe der Agrarwissenschaft wurde dabei weniger in der Sammlung und Aufbereitung von Erfahrungswissen gesehen, als in der Erzeugung von rationellem, systematischem Wissen, das gemäß der generellen modernen Überzeugung vor allem durch Abstraktion und Systematisierung der bisherigen Praxis überlegen sein solle: »Die wissenschaftliche Lehre setzt keine positiven Regeln [d.h. Erfahrungsregeln] fest, sondern sie entwickelt die Gründe, nach welchen man für jeden vorkommenden speziellen Fall - den sie scharf unterscheiden lehrt - das möglich beste Verfahren selbst erfindet « (Thaer 1809). Der moderne Glaube an eine wachsende gesellschaftliche Beherrsch- und Gestaltbarkeit von Natur durch die Erzeugung von wissenschaftlichem Wissen und durch Technologie hat die Industrialisierung der Landwirtschaft geformt und dabei im orientierenden Leitbild von »moderner Landwirtschaft « die Dualisierung von Natur und Gesellschaft vertieft.

7) So spricht die Bundesforschungsanstalt für Landwirtschaft (FAL) in ihrer Selbstdarstellung ohne Berücksichtigung der ausgelösten ökologischen, sozialen und ökonomischen Konsequenzen mit modernem Stolz davon, man habe »in den späten 70er Jahren eine Vervollkommnung der Produktionstechniken in allen [landwirtschaftlichen] Zweigen erreicht« vgl. <http://www.fal.de/de/about/entwicklung.htm> (eingesehen am 26.10.2004). 
Diese der rationalistisch und technologisch gedachten Modernisierung folgende Entwicklung fand ihren Höhepunkt in der zweiten Hälfte des letzten Jahrhunderts. Während die gesamtwirtschaftliche Bedeutung des Agrarsektors massiv abnahm - 1950 arbeiteten noch 23,3\% der deutschen Erwerbstätigen im Agrarsektor, 2003 nur noch 2,5 \% ${ }^{8}$ - nahm die Gesamtagrarproduktivität stark zu. Begleitet wurde dieser bis heute anhaltende Prozess einer gigantischen Ertragssteigerung bei gleichzeitig beständig abnehmender Zahl der in der Landwirtschaft Beschäftigten durch die Ökonomisierung, Professionalisierung und Verwissenschaftlichung der Landwirtschaft. Zudem setzt sich in der deutschen Agrarpolitik der Nachkriegsjahre in Auseinandersetzung mit liberalistischen Ideen ein starker staatlicher Protektionismus durch, der vor allem mit den »Eigengesetzlichkeiten der landwirtschaftlichen Urproduktion« (Heinrichsmeyer/Witzke 1991) gerechtfertigt wurde, also durch ihre von Immobilität gezeichneten Produktionsfaktoren und durch die Abhängigkeit von Naturgegebenheiten. Im protektionistischen Schwung degradierte die staatliche Regulierungsfreude die ehemals selbstbewusste Bauernschaft mehr und mehr zu stimmlosen Subventionsempfängern, zu Ausführenden der wechselhaften staatlichen Ordnungsansprüche, meist unter der bürokratischen Obhut der landesstaatlichen Ämter. Selbst die Europäische Bürokratie hat sich in hohem Maße an der Verwaltung des Agrarbereiches herausgebildet, die bis heute über die Hälfte des Haushaltes der Europäischen Union für sich beansprucht.

In gleicher Weise konnte das Bundesministerium für Landwirtschaft auch die entsprechenden (Ressort-) Forschungseinrichtungen unter staatlichem Dach bündeln, eine Konzentration, die offiziell mit dem aufgrund der kleinbetrieblichen Struktur des Agrarbereiches fehlenden Forschungspotential begründet wurde, tatsächlich aber in hohem Maße auch der Strategie des Ministeriums geschuldet sein dürfte, seine Stellung innerhalb der Regierung trotz abnehmender wirtschaftlicher Bedeutung des Agrarsektors durch Verwissenschaftlichung der Produktionsmethoden zu stärken (vgl. Hohn/Schimank 1990: 330f.).

Die wachsende Strukturierung und damit auch Abhängigkeit des Feldes durch staatlichbürokratische Institutionen einerseits, die zunehmende Verwissenschaftlichung und das damit verbundene Modernisierungsversprechen andererseits prägen bis heute den Alltag von Landwirten. Thaers Versprechen einer »Normalisierung « des Bauernberufes hat noch immer lockenden Bestand. »Der Bauer soll Unternehmer werden und der Bauernhof ein Betrieb [...] Dieses Versprechen hat jede Generation von jungen Bauern in der Nachkriegszeit neu bewegt « (Schmidt/Jasper 2001: 45). Besonders durch Mechanisierung, Technisierung und Chemisierung hoffte man die Abhängigkeit der Landwirtschaft von schicksalhaften Naturverhältnissen und von harter, menschlicher Arbeit im familiären Zwangsverband zu reduzieren und den Bauern zum ungebundenen, ökonomisch-rationalistisch statt traditionell denkenden Betriebsleiter zu machen. Die Industrialisierung der Landwirtschaft war mit dem Versprechen an die Bäuerinnen und Bauern verknüpft, endlich auch ihnen die Teilhabe an modernen Lebensformen zu ermöglichen, die für andere Teile der Gesellschaft längst selbstverständlich waren: Verkürzung der Arbeitszeit, Urlaub, freie Wochenenden, Bildung und Weiterbildungen sowie ein sicheres, gutes Einkommen.

Industrialisierung und staatlich massiv vorangetriebene Rationalisierung, in den letzten Jahren von der Öffnung für den Weltmarkt geprägt, griffen eng ineinander und verschafften der Landwirtschaft in nur wenigen Jahrzehnten ein neues, als »modern« apostrophiertes Gesicht: zu denken ist dabei auch an die systematisch durchgeführte Flurbereinigung (vgl. auch Scott 1999), an die späteren Energiesubventionen und Überschusskäufe sowie die Ausrichtung der staatlichen Forschung an Hochleistungszielen und schließlich mehr und mehr die nachgeschaltete Bekämpfung von symptomatischen Nebenfolgen. All dies setzte die Rah-

8) Vgl. Volks- und Berufszählung 1950 sowie Statistisches Bundesamt 2003. 
menbedingungen nicht nur für einzelbetriebliche Entscheidungen und einen langfristigen Trend des »Wachsens oder Weichens «, sondern auch für die daran anknüpfende, industrielle Nahrungsmittelproduktion, ${ }^{9}$ die preisaggressive Vermarktung und die ihr entsprechende Nachfragekultur. Bis spät in die 90er Jahre wurde von Seiten der Agrarwissenschaften die Steigerung der Produktivität als oberstes Ziel der Entwicklung im landwirtschaftlichen Bereich definiert (Kuhlmann 1996), obwohl Butterberge und Milchseen schon längst die Abkopplung der Produktion von tatsächlichen Bedürfnissen oder Vermarktungschancen im europäischen Raum aufzeigten.

Dass die industrielle Grundorientierung trotz beständig auftretender Lebensmittelskandale (DDT, Glykol, Dioxin etc.) lange Zeit relativ unangefochten weiter bestand, ist nicht zuletzt zwei Entwicklungsfolgen geschuldet: zum einen der Distanzierung der landwirtschaftlichen Produktion vom Rest der (agrarisch entfremdeten) Gesellschaft, zum anderen, und damit zusammenhängend, der hohen kulturellen und politischen Geschlossenheit des Agrarbereichs. Die Agrarproduktion war gesellschaftlich lange Zeit über bestimmte Bilder bäuerlicher, kleinstrukturierter Landwirtschaft repräsentiert, die mit der zunehmend industriell geprägten Realität in den meisten Teilen Deutschlands immer weniger übereinstimmten. Dies ermöglichte aber der agrarischen Interessenpolitik »eine Instrumentalisierung sozialmythischer Vorstellungen über die Landwirtschaft [..., die] von einem der Landwirtschaft weit entfernten Publikum deshalb dankbar aufgenommen werden, weil sie scheinbar Alternativen und Gegenbilder zu ihrer eigenen Lebens- und Arbeitsverhältnissen zeigen« (Rieger 1995: 38). Durch diese Vorstellungen im öffentlichen Bewusstsein wurde eine Auseinandersetzung mit der neuen »Realität « industrieller Landwirtschaft lange Zeit vermieden. Verbraucher wollten oft auch gar nicht so genau wissen, wo und vor allem wie die von ihnen billig gekauften Lebensmittel erzeugt werden. Gleichzeitig hatte die voranschreitende Industrialisierung der Landwirtschaft die Isolierung der Landwirte aus den sozialen dörflichen Kontexten zur Folge. Somit stieg ihre Abhängigkeit von den Agrarverbänden und damit vice versa auch die Unabhängigkeit der Verbände in der Bestimmung agrarpolitischer Leitlinien (vgl. ebd. 43).

Im Ergebnis führte diese Entwicklung zu einer hohen Geschlossenheit des Agrarbereiches. Der ständischen Interessenvertretung, dem Deutschen Bauernverband (DBV), gehören nach wie vor ca. 90\% der Bauern an, ein Organisationsgrad von dem andere Interessenverbände am Ende fester sozial-moralischer Milieus nur träumen können. Über Jahrzehnte war der Landwirtschaftsminister ein (Groß-)Bauer mit DBV-Hintergrund, so dass die deutsche Landwirtschaftspolitik auf Bundes- wie auf Landesebene »von Bauern für Bauern« gemacht wurde (vgl. Gerlach et al. 2005). Gleichzeitig ergibt sich aus der starken Verknüpfung von Bauernverband, Genossenschaften und Centraler Marketing-Gesellschaft der deutschen Agrarwirtschaft mbH (CMA) ein enges Netz persönlicher und fachlicher Beziehungen zwischen politischer Interessenvertretung und wirtschaftlicher Vermarktung, die schon vielfach Ziel von Kritik war. ${ }^{10}$ Ganz ähnlich sieht das Bild im Bereich von Agrarwissenschaft, wissenschaftlicher Politikberatung und Agrarpolitik aus: zwischen den Forschungsanstalten und den Ministerien gehen die typischen Karrierewege hin und her, kreuzen sich die Biographien in lange vorhersehbaren Mustern. Ein großer Teil der Akteure in Wissenschaft, Politik, Verwaltung

9) Wie schwer ein »Zurück« aus dieser Entwicklung ist, zeichnen Schmidt/Jasper (2001) am Beispiel der Fütterung von Kälbern mit Mutterkuhmilch nach: nachdem die jahrelange Praxis der Fütterung mit aus tierischen Fetten und Magermilchpulver bestehenden Ersatzstoffen nach BSE verboten wurde, wurde von wissenschaftlicher Seite mit aufwendigen Untersuchungen nachgewiesen, dass die Fütterung mit Mutterkuhmilch unbedenklich sei, also »wissenschaftlich gesehen nichts gegen die Natur [spricht] «(ebd. 36).

10) Ausdruck dieser engen Verknüpfung sind z.B. die oft in Personalunion geführten Ämter. Dr. Gerd Sonnleitner, der Präsident des DBV etwa hat über 30 Ämter und Funktionen inne (vgl. <http:// www.nabu.de/landwirtschaft/datenbank.htm> eingesehen: 03.12.2004). 
und Verbänden kennt sich bereits aus dem Agrarstudium. Das Ergebnis ist insgesamt ein engmaschiges Netzwerk aus Forschung, Politik, Verwaltung, Unternehmern und Vertretern der verschiedenen Agrarverbände, dem es lange Zeit gelungen ist, Ambivalenzen und konkurrierende Deutungs- und Bewertungsansprüche klein zu halten. Die zunehmenden Widersprüche innerhalb des Bereiches Landwirtschaft, etwa zwischen großen und kleinen Betrieben, aber auch zwischen unterschiedlichen Erwartungen und Ansprüchen verschiedener Akteursgruppen der Wertschöpfungskette sowie zwischen den verschiedenen Teilsystemen von Politik, Wissenschaft, Wirtschaft und Familie blieben so lange Zeit latent, ohne die scheinbare Erfolgsgeschichte der modernisierten Landwirtschaft in Frage zu stellen. Es schien daher nicht verwunderlich, dass gerade jener Bereich, auf den die frühen Schriften der Umweltbewegung zentral zielten (vgl. Carsons 1962, Meadows 1972, Merchant 1987), selten Ziel scharfer umweltpolitischer Ansprüche oder Gegenentwürfe wurde. Selbst der Nachhaltigkeitsdiskurs wendete sich bis Mitte der 1990er Jahre dem Problembereich von Landwirtschaft und Ernährung in Deutschland nur zögernd und behutsam zu.

Tatsächlich hat es dennoch im Agrarbereich marginalisierte Abweichungen von dieser dominanten Orientierung gegeben, alternative Leitbilder, widersprechende Zielvorstellungen und konkurrierende Rahmungen - auch bei den Bauern. Allen voran profilierte sich in der Nische der so genannte ökologische Landbau mit seinem Leitbild einer naturgerechten, nichtchemischen und den Betrieb holistisch als »lebendigen Organismus« begreifenden Landwirtschaft. Anders als die konventionelle Landwirtschaft ist der Ökolandbau kein Produkt von Wissenschaft und Agrarindustrie, sondern folgte einer sehr viel enger an Erfahrungswissen und Praxis ausgerichteten Innovationslogik. »Obwohl Wissenschaftler und Philosophen [...] die Konzepte und Grundideen biologischer Produktion vorlegten, waren es vor allem erfahrene Landwirte und Gärtner, welche die Methoden der biologischen Landwirtschaft entwickelten und voranbrachten « (Niggli 1999: 38). Insofern entwickelte er sich abseits der üblichen Arenen. Eng verbunden mit alternativen Weltanschauungen, den Lebenskonzepten einer »Gegenbewegung « und oftmals postmateriellen Wertvorstellungen schuf er sich über die Jahre eine - in sich durchaus heterogene - institutionelle >Parallelwelt< von Erzeugerverbänden, Veröffentlichungsorganen und Vermarktungsstrukturen. Spätestens im Zusammenhang mit dem Aufstieg der Neuen sozialen Bewegungen meldeten sich diese »Alternativen« zusammen mit anderen, von Agrar-Mainstream und Bauernverband enttäuschten Akteuren auch politisch mehr und mehr zu Wort. Die so entstehende »Agraropposition« aus Ökolandbau, Vertretern bäuerlicher Landwirtschaft, regionalen Bauernzusammenschlüssen, Eine-Welt-Gruppen und Natur- und Umweltschutzverbänden ${ }^{11}$ drängte auf einen generellen Richtungswechsel in der Agrarpolitik hin zu einer Förderung naturverträglicher und extensiver Landwirtschaft. Dennoch blieben diese Gegenkonzepte äußerst marginalisiert. Sie wurden in den entscheidenden Arenen nicht nur kaum zur Kenntnis genommen, sondern als Abtrünnige oder Spinner, als »fachlich wenig qualifizierte >Neo-Alternative< [...] mit dem für Konvertiten stets kennzeichnenden dogmatischen Eifer« (Ziche/Kromka 1982: 109) ausgeschlossen.

Aber auch innerhalb des Agrar-Mainstreams waren über die Jahre Brüche im eindeutig auf Technisierung und Intensivierung orientierten Leitbild zu erkennen. Im Zuge der Umweltdebatte mussten zunehmend gesellschaftliche Ansprüche nach einer umwelt- und tierfreundlicheren Landwirtschaft abgewehrt werden. Der Bauernverband etwa stellte erst jegliche negativen Umwelteffekte der Landwirtschaft in Abrede, ging dann dazu über, diese einzelnen >schwarzen Schafen< in der Landwirtschaft zuzuschreiben, um schließlich, als sich breite ökologische Folgen nicht mehr leugnen ließen, die schlechte ökonomische Lage der Land-

11) Ende der 80er Jahre schlossen sich Verbände aus den genannten Bereichen unter dem Namen »Dachverband der Deutschen Agraropposition« zusammen (vgl. Thielen 1991). Die Bezeichnung ist also dem Feld entnommen. Heute nennt sich diese Vereinigung »AgrarBündnis«. 
wirtschaft als Grund anzuführen (vgl. Heinze/Voelzkow 1994: 167f.). Auch in der Agrarwissenschaft herrschte lange die Auffassung vor, dass es »ja nicht angehen kann, dass zwanzigtausend Wissenschaftler in Europa von einer Handvoll Umweltaktivisten gehänselt werden«, wie ein Wissenschaftler stellvertretend formulierte (zit. nach Pior 2000: 78). In den 90er Jahren hat sich dann jedoch spürbar eine langsame Öffnung des Selbstverständnisses auch der konventionellen Agrarwissenschaft gegenüber den Anliegen von Umwelt- und Naturschutz ergeben (vgl. Mante 2005). So ist auf der Ebene der Selbstthematisierung eine langsame Gewichtsverschiebung weg von der Zieldefinition »Steigerung der Gesamtagrarproduktion « (Kuhlmann 1996) hin zur »Modernisierung und Nachhaltigkeit von Land- und Forstwirtschaft, einschließlich ihrer multifunktionalen Rolle für die Gewährleistung von ländlicher Entwicklung und der Förderung ländlicher Gebiete« (BMBF 2002) zu erkennen. Die Gründe dafür sind sicherlich vielschichtig und weder als radikaler Bruch mit Traditionen noch als reine Rhetorik zu identifizieren. Viel mehr scheinen sie aus einer ambivalenten Mischung von Reaktionen auf EU-Umweltprogramme und öffentliche Diskurse zu bestehen, aber auch aus der wachsenden Billigung einer Notwendigkeit nachhaltigerer Entwicklungswege sowie der reflexhaften Suche nach neuen Legitimationsmustern vor dem Hintergrund entwerteter Routinen und Institutionen. Ein wichtiger Grund für das Aufgreifen der Umweltschutzthematik im Agrarbereich liegt sicherlich auch in der Hoffnung, durch den Verweis auf die Multifunktionalität ${ }^{12}$ der Landwirtschaft trotz der im Zuge der WTO ausgehandelten Liberalisierung der Agrarmärkte staatliche Subventionen weiterhin zu rechtfertigen.

Trotz langer institutioneller und kultureller Geschlossenheit im Schutzraum öffentlicher Gleichgültigkeit, trotz und neben der fraglosen Hegemonie des industriellen und auf Produktionssteigerung ${ }^{13}$ ausgerichteten Entwicklungspfades und trotz der vermeintlichen Eindeutigkeit und Alternativlosigkeit der zugehörigen Leitbilder und Zielvorstellungen hatte im Agrarbereich schon vor den Skandalen um BSE und MKS ein langsamer, ambivalenter, gebrochener und uneindeutiger Wandel begonnen, auf den die dominanten Akteure teilweise mit Abwehr, teilweise mit Integration und Reformulierung - etwa in Form einer Ökonomisierung des Ökologieproblems (vgl. Heinze/Voelzkow 1994: 167f.) - reagiert haben. In wie weit diese Veränderung der Legitimationsmuster auch für die Praxis, etwa der Agrarwissenschaften, relevant geworden ist, ist allerdings hoch umstritten (vgl. Bundesamt für Naturschutz 2002, Isermeyer 2003, Ober 2004).

\section{Die institutionelle Verarbeitung von Risiken, Folgenreflexion und Agrar- wende}

Dennoch scheint die Wucht, mit der die Lebensmittelskandale um BSE, MKS und Nitrofen den Agrarbereich aufgewühlt haben, überraschend. Anders als bei früheren Risikoskandalen gelang es den klassischen Institutionen des Agrarbereiches nicht, die Ereignisse in den Jahren 2000/01 als unerwünschten Störfall eines ansonsten unproblematisch laufenden Normalbetriebs der Lebensmittelerzeugung dazustellen. Minister mussten zurücktreten, der Rindfleischmarkt brach über Nacht zusammen, die öffentliche Verunsicherung fand ihren Ausdruck in Überschriften wie »Was können wir überhaupt noch essen?«. Zwar gab es auch hier die üblichen Versuche, die Gefährdung symbolisch herunter zu spielen: Bspw. griff der britische Landwirtschaftsminister John Gummer eine bereits in den 80er Jahren gerne ge-

12) Das Konzept der Multifunktionalität betont, dass Landwirtschaft gleichzeitig zu mehreren ges. Funktionen einen Beitrag leistet, also neben der Lebensmittelerzeugung auch dem Schutz der Umwelt und der Entwicklung ländlicher Gebiete dient.

13) Auch heute wird von Seiten der Agrarwissenschaft darauf hingewiesen, dass angesichts des Wachstums der Weltbevölkerung die Steigerung der Produktion das zentrale Ziel der Innovationen im Agrarbereich bleiben müsse (vgl. Isermeyer 2001). 
wählte Strategie der symbolischen Exemplifizierung durch »Selbstversuch unter Zeugen« auf, die an die Anfänge der experimentellen Wissenschaft erinnert (Latour 1990). Er aß zusammen mit seiner kleinen Tochter demonstrativ Beef-Burger. Andere suchten in mehr Kontrolle und verschiedenen Formen der Grenzziehung neue Sicherheit (bspw. Trennung von als riskant eingestuftem Separatorenfleisch von als sicher apostrophiertem Fleisch ohne Kontakt zum zentralen Nervengewebe sowie junger von alten und britischer von europäischen, ja sogar deutschen von bayerischen Rindern; vgl. Tacke 2001). Jedoch führten diese Taktiken auf lange Sicht zur umso heftigeren Delegitimierung der bisherigen institutionellen Routinen und Sicherheitsversprechen. Unwiederbringlich schien das Konsumentenvertrauen verloren, gerieten Veterinäre, Politiker, Bauernverband als Hüter von Sicherheit, Wohlstand und Ordnung unter den Dauerverdacht, Gefahren in die Welt zu setzen, zu verharmlosen und zu vertuschen, folgenblind und ohnmächtig geradezu mit Scheuklappen den entwerteten Kontrollund Wettbewerbsversprechungen vergangener Jahre nach zu laufen. Andererseits haben sich die mit der Krise ausgelösten Turbulenzen langsam wieder gelegt, so dass es zunächst vor allem eine empirische Frage ist, in wie weit und wie tief greifend die tradierten Institutionen und Selbstverständnisse im Agrarbereich erschüttert und grundlegend herausgefordert sind oder ob eine Rückkehr zum business as usual, im Fachjargon eine »funktionale Anpassung «, möglich ist.

Zuerst möchten wir herausstreichen, dass Institutionen im soziologischen Sinne vor allem durch ihre Beständigkeit gekennzeichnet sind. Sie entfalten erst dann handlungsorientierende, normbildende und entscheidungsentlastende Kräfte, wenn es ihnen gelingt, über kurzfristige Bedeutungsverschiebungen und Krisen hinweg Handeln und Entscheiden kollektiv verbindlich anzuleiten. Institutionen schaffen die Voraussetzung eines koordinierten Zusammenlebens und bieten Orientierung in einem überkomplexen gesellschaftlichen Feld, wenn sie als kontinuierliche Regelsysteme sozialer Interaktion auftreten (vgl. Giddens 1988, Göhler 1994). Sie werden nur fraglos reproduziert, wenn sie sinnstiftende Wirklichkeitsdeutungen setzen und auf Handlungsprobleme angemessene Problemlösungsstrategien normativ definieren. Mary Douglas (1991) hat zudem herausgestellt, welche Bedeutung für diese Funktionen und das »Denken der Institutionen « klassifizierende, meist dichotom strukturierte Schemata haben, innerhalb derer der Horizont gesellschaftlich akzeptierter Entscheidungsund Begründungsmodi vermessen und hierarchisch geordnet wird. ${ }^{14}$ Werden solche Duale (bspw. zwischen Natur und Gesellschaft, Wissenschaft und Politik, Landwirtschaft und Industrie, oder einfach »oben« und »unten«, »Zentrum« und »Peripherie«) im Rahmen von Risikodiskursen in Frage gestellt oder anhand krisenhaft erzwungener Prozesse einer nach innen gewendeten Reflexion »ausgehebelt «, verlieren die Institutionen jene Leitunterscheidungen, anhand derer sie normieren und Sicherheiten schaffen. Sie werden blasser und büBen ihre verbindlich standardisierende, Gesellschaften strukturierende Kraft ein - bis entweder neue Grundkategorien an die Stelle der alten treten oder sie von Ausnahme zu Ausnahme zerfressen werden und zerfallen. Können Institutionen die alltäglichen Handlungs- und Entscheidungsprobleme nicht mehr strukturieren und werden deshalb durch das alltagspraktische Handeln sozialer Akteure nicht mehr reproduziert und in ihrer Geltung immer wieder bestätigt, »trocknen sie aus und werden unplausibel« (Offe 1989: 772). Was aber passiert, wenn Institutionen mit vielfachen Entgrenzungen durch Pluralisierung konfrontiert sind und eingestandenermaßen vor dem Hintergrund von Unsicherheit und Ambivalenz operieren?

14) Die universalisierende Beschreibung von Mary Douglas mag bereits in sich der typisch einfach-modernen Grundüberzeugung geschuldet sein, die ein Denken in Ambivalenzen und Unsicherheiten per se als vormodern ablehnt und stattdessen geordnet kategorisieren möchte. Nichtsdestotrotz sensibilisieren ihre Fallstudien und Überlegungen für die Schwierigkeiten, die in Institutionen aufbrechen, wenn die dichotome Strukturierung und orientierende Klassifikationen aufbrechen, wegbrechen, pluralisiert oder zersetzt werden. 
Dies war immerhin die Situation der agrarwissenschaftlichen und agrarpolitischen Institutionen seit der Jahreswende 2000/2001.

Werfen wir diesbezüglich einen genaueren Blick auf die Prozesse sowohl des programmatischen »Politikwechsels« und seiner Gelegenheitsstrukturen als auch der eher schleichenden, institutionellen Transformation im Gefolge der BSE- und MKS-Krisen: inwiefern interferieren Funktionskrise und Legitimationskrise im Gefolge von Nebenfolgen und Nebenfolgen der Nebenfolgenreflexion.

\section{Öffentliche Sichtbarkeit der scheiternden Sicherheitsversprechen}

Die Vorgänge um BSE haben das Scheitern der bestehenden Einrichtungen von Risikoabschätzung und -management sozusagen unter öffentlicher Beobachtung vorgeführt. Erst versuchte die britische Regulierungspraxis jahrelang, Unsicherheiten und Unwissen zu vertuschen und den Anschein der Kontrollierbarkeit und vor allem Sicherheit zu erzeugen. Wissenschaftliche Warnungen vor einem möglichen Zusammenhang zwischen der bei Rindern vermutlich durch eine riskante Form der Tiermehlverwendung in Futtermitteln ausgelösten Seuche - die große Ähnlichkeiten mit Scrapie, einer bei Schafen bekannten transmissiblen spongiformen Enzephalophatie (TSE) aufwies - und der menschlichen TSE-Variante, der Creutzfeldt-Jakob-Krankheit (CJD), hat es von Anfang an gegeben (vgl. hier und im Folgenden: Gee/Guedes/Vaz 2004: 181ff.). Die Strategie der Behörden aber war davon geprägt, wirtschaftliche Nachteile für die britische Lebensmittelindustrie sowie große Ausgaben für die öffentliche Hand zu vermeiden. Wie bei vorhergehenden Lebensmittelskandalen auch schon ging es darum, einer folgenschweren Konsumentenverunsicherung unbedingt vorzubeugen - zumindest solange wissenschaftliche Beweise nicht eindeutig vorlagen - und grundsätzlich jeden Anschein zu vermeiden, british beef, ein mit Wohlstand assoziiertes Nationalgericht, könne ein Gesundheitsrisiko sein. ${ }^{15}$ So wurden bestimmte Experten, etwa vom britischen Health Laboratory Service, aus den entsprechenden Beratungsgremien ausgeschlossen, um keinerlei Verbindung zwischen der Rinderseuche und einer möglichen Gefährdung der menschlichen Gesundheit denkbar zu machen. Diese Strategie wurde im Laufe der Wochen, Monate und Jahre immer mehr zum Selbstläufer. Kostengünstige und relativ einfache Vorsorgemaßnahmen unterblieben, weil man fürchtete, damit indirekt zuzugeben, dass überhaupt ein Handlungsbedarf bestehe. Auch die Regulierungspraxis folgte der modernen Logik des Entweder-Oder: entweder gilt BSE als wissenschaftlich bewiesene Gefährdung für die menschliche Gesundheit, und erst dann wären staatliche Regulierungen notwendig, oder aber BSE ist eine Tierseuche, und dann muss alles vermieden werden, was die fundamentale Grenzlinie zwischen Agrarskandal und Gesellschaftskrise durchbrechen könnte. Für Unsicherheiten bzw. Ambivalenzen war damit kein Platz. Umso heftiger waren schließlich die Reaktionen, als die Möglichkeit eines Zusammenhangs zwischen BSE und der CJD-Variante nicht mehr von der Hand zu weisen war und konkurrierende Deutungen täglich an öffentlichem Gewicht gewannen.

Auch wenn Deutschland insgesamt flexibler und schneller reagiert hat (Böschen/Viehöver/Zinn: 2002), so wurde auch hier bis zum Jahresende 2000 weitgehend eine Strategie der

15) Die sehr rigiden und schematischen Bemühungen der Abwehr jeder Form von möglicher Kritik an der intensivierten Rinderzucht werden erst vollständig verständlich, wenn man berücksichtigt, dass bereits seit längerem ein an den Grundsätzen der westlich-industriellen Lebensweise rüttelnder Gegendiskurs gerade Rindfleisch und seine Produktionsbedingungen in den Mittelpunkt seiner Fundamentalkritik stellte und deutlich auf die nicht nur ökologischen Konsequenzen aufmerksam machte (vgl. dazu Rifkin 2001). 
Problemnegierung verfolgt. ${ }^{16}$ Das Auftreten des ersten BSE-Falles eines in Deutschland geborenen und von deutschen Rindern abstammenden Tieres im November 2000 war denn auch nicht wegen einer davon ausgehenden Zunahme der Gefährdung skandalös - schließlich hatte es bereits vorher Fälle von BSE-Rindern in Deutschland gegeben und man stand auch kurz davor, den Import britischen Rindfleisches nach Deutschland wieder zuzulassen. Vielmehr verkörperte dieses Rind nun auch in Deutschland das endgültige Scheitern eines modernen Sicherheitsversprechens, das an die klassischen Risikoinstitutionen geknüpft war: an die nationale Betrachtung, an die Fundierung durch wissenschaftlich legitimierte Beweise, an die staatlich-politische Kontrolle durch Bundesbehörden als Garanten von sozialer und wirtschaftlicher Sicherheit. Da einfache Strategien der Krisennegierung - Verzeitlichung, Naturalisierung, Verharmlosung - damit endgültig unbrauchbar waren, folgten auch in Deutschland massive Umwälzungen: eine »Städterin« ohne »Stallgeruch« wurde zur Ministerin für Agrarfragen berufen, der Zuschnitt des Ministeriums verändert, neue Gesetze und Verordnungen in ungesehener Eile verabschiedet, zwei neue Behörden ${ }^{17}$ für Risikomanagement gegründet.

Diese typischen Formen von akutem Krisenmanagement, Entfernung belasteter Namen, Verschiebung von Zuständigkeiten, Ausdehnung von Kontrolle sowie Einrichtung neuer Behörden, sind zentral von dem Motiv geprägt, eine strukturell riskante Krise zu überwinden, um so rasch wie möglich zum Normalbetrieb zurückzukehren (vgl. 't Hart/Boin 2001). Sie fallen unter den modernen Modus der funktionalen Anpassung an eine Funktionskrise. Mit der Ankündigung eines »tief greifenden«, politischen »Richtungswechsels« wurde angesichts der öffentlichen, massenmedial verstärkten Legitimationskrise der Modus der reinen Bewältigung jedoch auf programmatischer Ebene bereits zu Gunsten eines folgenreichen Politikwechsels - teilweise unter Einbezug bestehender Bezugsrahmen - überschritten. Ganz im Sinne der Theorie reflexiver Modernisierung versucht die propagierte »Agrarwende«, »the long shadow of post-crisis politics« (ebd.) mitzugestalten. Im Bewusstsein, dass unter öffentlicher Beobachtung jede, auch strukturelle, Nachbesserung inhärent der Entwertung absoluter Kontroll- und Objektivitätsansprüche gleichkommt und gerade die Heterogenität der betroffenen Dritten und deren Folgenwahrnehmungen für eine nicht zu begrenzende Politisierung und Nebenfolgendynamik sorgen wird (vgl. Dewey 2001), wurde von politischer Seite selbstbewusst die Reflexion auf die bis dato fraglosen Rationalitätsgrundlagen im Agrarbereich in Angriff genommen. Alle beteiligten Akteure betrachteten in der Folge gemeinsam mit einer breiten, nun weit über die Fachkreise hinausgehenden Öffentlichkeit die Ambivalenz und Kontingenz von als sicher und zwangsläufig dargestellten Entwicklungen

16) Als die nordrhein-westfälische Umwelt- und Agrarministerin Bärbel Höhn 1998 eine Studie mit den neu entwickelten BSE-Schnelltests durchführen ließ, wurde ihr mit Verweis auf Deutschlands »Freiheit von BSE« die Verschwendung von Steuergeldern vorgeworfen. Ein hochrangiger Politiker, den Kerstin Dressel (2001) in ihrer BSE-Studie befragte, charakterisiert diese Haltung treffend: »Und die sagten: Wir sind uns sicher, dass wir BSE-frei sind, also gucken wir nicht nach [...] das ist ein Verhalten wie [in] Brechts Galileo Galilei. Die Bischöfe, die durchs Fernrohr gucken sollen, um das Sonnensystem zu erfassen, sagen: nein, wir gucken nicht durch, denn es könnte sich herausstellen, dass Galileo recht hat $[. .$.$] so verrückt war das.«$

17) Auf Empfehlung des vom Bundeskanzler initiierten sog. »Von-Wedel-Gutachtens« (2001) zu den Schwachstellen in der Organisation des gesundheitlichen Verbraucherschutzes wurde die scheinbar eher wissenschaftliche Risikobewertung und -kommunikation vom eher politischen Risikomanagement organisatorisch getrennt. Ersteres sollte das neu gegründete Bundesamt für Risikobewertung (BfR) übernehmen, letzteres wurde dem Bundesamt für Verbraucherschutz und Lebensmittelsicherheit (BVL) zugeschrieben. Böschen et al (2002: 24) kritisieren zu recht, dass diese Konstruktion »die Idee einer klassischen Arbeitsteilung zwischen Wissenschaft und Politik « festschreibt und somit eine institutionelle Lernperspektive für den produktiven Umgang mit Ungewissheit und Nichtwissen vermissen lässt (ebd. 25). 
im Agrarsektor: im Vergleich zur BSE-Bewältigung in Großbritannien und den Niederlanden schien eine »reflexive Risikopolitik« in Deutschland in diesen Tagen greifbar, verlor sich aber in dem Maße, wie Unsicherheiten und Ambivalenzen auch der propagierten Alternative nicht transparent gemacht wurden (vgl. Oosterveer 2002: 224).

Die bis dato an Agrarfragen eher desinteressierte Bevölkerung erschrak ob der gesellschaftlich ausgeblendeten Praktiken im Umgang mit Nutztieren. Sie fühlte sich einer radikalen Verunsicherung und auch Politisierung ausgesetzt, wie sie typischer Weise aus dem Zusammenbruch des alltagsentlastenden Vertrauens in Expertensysteme und insbesondere aus der Infragestellung unhinterfragter Basisprämissen der modernen Lebensführung erwächst (vgl. Giddens 1991). Mit der öffentlichen Dramatisierung der Krisen, die nicht nur bildlich vielfach in die Nähe einer »modernen Apokalypse « gerückt wurden, wurden vor allem jene Rahmungen und Deutungsmuster der modernen Gesellschaft prekär, die ihr Naturverhältnis absichern (vgl. Viehöver/Wehling/Keller in diesem Heft). Es schien, als würden sich die Gesellschaftsmitglieder erst im Anblick blutiger Ställe und massenhaft hingerichteter, brennender Tierleiber jenes radikal instrumentellen Naturbegriffs bewusst, der konstitutiv für die Moderne ist und deren carnivore »conscience collective « im Sinne Durkheims bestimmt (vgl. Eder 1998: 112). Die antithetische Architektur des modernen Natur- und Gesellschaftsbegriffs (vgl. Kropp 2002) und die darin verborgene, mitunter grausame Prämisse des industriellen Wohlstands schimmerte wenige Wochen auch im ignoranten Konsumentenbewusstsein auf, verknüpfte das Leiden der Kreatur wie der Bauern mit den je eigenen Ernährungspraktiken und der darin stabilisierten, moralischen Ordnung. Das mediale Rütteln an den fundamentalen Prämissen moderner Lebensführung bewegte die Fleischindustrie, die Feuilletons und die Politiker gleichermaßen.

Diese reflexiv-fundamentale Erschütterung traf auf eine »politische Gelegenheitsstruktur«: Mit Bündnis90/Die Grünen war eine Partei an der Regierung beteiligt, die seit jeher in einem engen Verhältnis zur der eher marginalisierten >Agraropposition « stand. Für diese Kräfte und weitere kritische Anspruchsgruppen (NGOs etc.) öffnete sich mit den Skandalen um BSE und MKS ein Einfallstor, um ihre Kritik an den industrialisierten Verhältnissen im Agrarsektor und an der damit verbundenen Abhängigkeit der Landwirtschaft von Chemieund Lebensmittelindustrie zusammen mit der Forderung nach einer ökologischeren, »anderen « Landwirtschaft mit Nachdruck auf die politische Agenda zu setzen. Auch deshalb konnten die Skandale und ihre öffentliche Wahrnehmung erhebliches Veränderungspotential entwickeln, weil sie auf eine besondere Akteurskonstellation trafen, in der es Interesse und Bereitschaft gab, die Impulse aufzugreifen, um die bestehenden Strukturen in Frage zu stellen und insbesondere die alten Rücksichten auf agrarpolitische Interessensverbände und Einflussstrukturen aufzugeben.

\section{Nebenfolgencharakter}

Neben der öffentlichen Sichtbarkeit der Scheiterns der industriemodernen Organisationen und Institutionen, der daraus zunächst erwachsenden »Gegenöffentlichkeiten« und schließlich ihrem Niederschlag in institutioneller und kognitiver Pluralisierung und Entgrenzung verstärkte ein zweiter, typisch risikogesellschaftlicher Aspekt die besondere Wirkmächtigkeit der Agrarskandale: sie ließen sich klar als Folgen vorhergehender politischer Entscheidungen deuten, erschienen nicht als Folgen von Missbrauch oder »krimineller Machenschaften«. Fraglos sind BSE und MKS die nicht intendierten Folgen des »Normalbetriebs«, eine Einsicht, die der um sich greifenden Nebenfolgendynamik in Wissenschaft und Politik die Türen öffnet.

Bruno Latour (2004) hat dieses Charakteristikum sehr eindrücklich für die Krise um das Wiederauftreten der Maul- und Klauenseuche in Großbritannien im Sommer 2001 nachgezeichnet. Er argumentiert, dass es sich hierbei nicht um die Bekämpfung einer überraschend 
und unvermeidlich ausbrechenden Seuche handelte, sondern um »die unerwünschten - wenn auch vorhersehbaren - Folgen eines Experiments im gesamteuropäischen Maßstab« (ebd. 185). 1992 wurde die bisherige Praxis, die Tiere gegen MKS zu impfen und damit die Seuche zwar nicht auszurotten aber zumindest zurück zu drängen, EU-weit verboten. Grund dafür war, dass geimpfte Tiere - die den Erreger ja aufwiesen - nicht in Länder wie die USA oder Japan exportiert werden konnten. Stattdessen führte Brüssel die Eradikations-Regelung ein, die eine Tötung und Beseitigung von erkrankten und gefährdeten Tieren vorsah. Die stolze, sehr moderne Hoffnung war, Europa im Rahmen flächendeckender Kontrollbemühungen seuchenfrei zu bekommen - angesichts des weltweiten Waren- und Personenverkehrs und der leichten Übertragbarkeit von MKS durch eine Vielzahl von belebten und unbelebten Zwischenträgern ein - milde ausgedrückt - sehr ambitioniertes Experiment. Dabei ist nicht entscheidend, ob die Fortsetzung der Impfung »besser« gewesen wäre. Entscheidend ist, dass der neuerliche Ausbruch der Seuche als Nebenfolge von Entscheidungen auftrat, die aus der Jahrhunderte alten »Gefahr«, mit der Generationen von Bauern gelebt hatten, plötzlich ein »Risiko« (Luhmann 1991) gemacht hatten.

Das MKS-Risiko fiel damit auf jene Organisationen und Regulierungsanstrengungen zurück, die eigentlich mit der Beseitigung von Risiken beauftragt waren. Dennoch konnten jene sich aber ihres Auftrages auch nicht einfach entledigen, sondern standen und stehen nach wie vor vor der Herausforderung, Risiken zu vermeiden, Sicherheit zu schaffen und damit ihre Funktionsfähigkeit aufrecht zu erhalten. Institutionen sind darauf ausgelegt, sich selbst zu stabilisieren - und dies insbesondere Institutionen der politischen Entscheidungsfindung in pluralistischen Demokratien (vgl. Boin/'t Hart 2000). Diese Eigenlogik der Stabilisierung kann zu einer Abwehr von Verantwortung und Zuschreibung der Nebenfolgen führen, die mit der Zeit selbst zur Eigendynamik wird: Fassaden werden aufrechterhalten und verteidigt und umso mehr Energie in die Verteidigung fließt, umso wichtiger wird es, die Fassaden um ihrer selbst willen aufrecht zu erhalten. Damit erscheint jede Reflektion des eigenen Handelns und der zugrunde liegenden Orientierungen und Zielsetzungen nun als gefährdend. Gerade diese Eigendynamik entkernt letztlich die Institutionen von innen heraus, macht sie unflexibel und starr und lässt sie immer weniger passgenau zu einer sich verändernden Realität werden: in der Folge orientiert sich das Handeln mehr und mehr am »Als ob« (Ortmann 2004) und macht gerade durch die beinahe zwangsläufig betriebene Entkoppelung von talk und action die institutionellen Grundorientierungen verfügbar. Wird die Entkopplung offenbar, fallen die Fassaden schließlich in sich zusammen.

Gleichzeitig gewann im Agrarbereich nach der Erschütterung durch BSE und MKS die Reflexion der Nebenfolgen, die innerhalb der Institutionen peinlich vermieden wurde, außerhalb, in anderen, auch neu entstehenden Arenen (vgl. Viehöver et al. 2004: 78) zunehmend an Bedeutung. Die medienvermittelte Vielfalt von Problem- und Kriseninterpretationen sowie die Konjunktur von alternativen Problemlösungsangeboten trat schließlich eher von außen an die bisherigen Akteursnetzwerke heran und führte zu einer rasch wachsenden Delegitimierung derjenigen, die auf Eindeutigkeit und kontrolliertes Management durch bestehende Organisationen und im Rahmen bisheriger Institutionen gepocht hatten. Die agrarwissenschaftliche und agrarpolitische Fachöffentlichkeit fand sich in einem vielstimmigen Kanon von Ansichten, Forderungen und Interpretationen wieder, indem sie die Definitionshoheit schnell verloren hatte. Verstärkt wurde diese Entgrenzung bisheriger Sprecherrollen durch die defizitäre bis fehlende Selbstthematisierung und Selbstproblematisierung bei den etablierten Akteuren. So sah man z.B. im Mainstream der Agrarwissenschaften zwar die Notwendigkeit, den Zielkatalog der Forschung an die »aktuellen gesellschaftspolitischen Herausforderungen« (Initiativkreis Agrar- und Ernährungsforschung 2002) anzupassen, jedoch wurden die Forschungspraktiken, Methoden und Paradigmen sowie die eigene Rolle in der bisherigen Entwicklung und ihren krisenhaften Ausläufern kaum problematisiert. Dieses 
Feld überließ man vollständig der Agraropposition, die auch flugs entsprechende Veranstaltungen durchführte und Foren eröffnete. ${ }^{18}$ Die Nebenfolgen hatten damit die Institutionen des Agrarbereiches endgültig erreicht.

Während die nachgezeichnete Sichtbarkeit des Scheiterns vor allem die Interpretationsfolie für den schnellen, in Reaktion auf Skandale antretenden politischen Wende-Anspruch abgibt, berücksichtigt der Fokus auf die fundamentale Erschütterung durch den Nebenfolgencharakter den damit ausgelösten langsameren und versteckteren Wandel von Bezugsrahmen sowie seine Einbettung in einen übergreifenden Prozess der Pluralisierung und Entgrenzung.

Tatsächlich müssen beide Perspektiven verzahnt werden, um die Chancen und Restriktionen des institutionellen Wandels im Gefolge der großen Krisen angemessen einzuschätzen. So ließ sich bspw. die »Agrarwende « als Reaktion auf die Krisen der Intensivproduktion nur deshalb formulieren, weil es alternative Problemlösungsangebote aus der Agraropposition sowie dem Diskurs zu nachhaltiger Landwirtschaft überhaupt gab und weil diese Diskurskoalitionen nicht nur Konzepte, sondern auch institutionelle Denkweisen, Netzwerke und praktische Erfahrungen vorzuweisen hatten. Damit Nebenfolgen im Rahmen des Nebenfolgentheorems Transformation und Reflexion oder gar einen reflexiv-modernen Wandel generieren bzw. befördern können, bedarf es diskutabler Alternativen auf der kognitiven wie auf der Handlungsebene. Andererseits trafen die propagierten Wende-Konzepte auf wochenlange, weitgehend folgenblinde Stabilisierungsbemühungen, in deren Rahmen die Bewahrung der institutionellen Fassaden gegen alle konkurrierenden Deutungsansprüche betrieben wurde. Diese Gegenkräfte bestehen, auch weil bis heute eine Behörde undenkbar ist, die offen einräumt, ihre Entscheidungen auf Nichtwissen zu begründen (vgl. auch Mayntz 1999) oder über keine identifizierbaren Entscheidungs- und Begründungsleitlinien zu verfügen, orientierungslos zwischen wissenschaftsbasierten und wissenschaftsexternen Problemlösungsangeboten zu wanken und ratlos angesichts von Komplexität und Ambivalenz zu sein. Wie oben erwähnt (vgl. Fußnote 5) ist ein postmodernes »anything goes « mit der modernen Begründungs- und Legitimierungsnotwendigkeit, insbesondere in der Administration, nicht vereinbar. Nicht zuletzt durch die institutionellen Funktionsnotwendigkeiten der Normalisierung und Stabilisierung haben sich Feindbilder verschärft und Gräben vertieft, bspw. zwischen den neuen »Vorzeigekindern« des Ökolandbaus und all jenen, die sich - respektive vergeblich - weiterhin bemüht hatten, den status quo zu rechtfertigen und zu verteidigen.

Verbindet man daher die eher politikwissenschaftliche Diskussion des Politikwechsels (vgl. Feindt/Ratschow 2003, Mol/Bulkeley 2002) mit der eher gesellschaftstheoretischen Betrachtung von reflexiver Modernisierung und dem Wandel der Bezugsrahmen (vgl. Oosterveer 2002, Hajer 2004), bekommt man ein neues Verständnis von den transformativen Bedingungen des inkrementellen und gebrochenen Wandels von alten zu neuen Institutionen. Ulrich Beck und Christoph Lau (in diesem Heft) gehen davon aus, »dass alte Strukturen (Basisinstitutionen) nicht einfach [...] ersatzlos erodieren und selbst nach dem Prinzip des >EntwederOder < ersetzt werden. Wäre dies zutreffend, gäbe es also einen >reinen Typus< des Neuen, so wäre dieser selbst noch in den Aporien der Eindeutigkeit befangen.« Vielmehr müsse auch »das Neue selbst nach dem Prinzip des 〉Sowohl-als-Auch $<$ gedacht werden« (ebd.), trete also nicht in »reiner Form auf, sondern in unterschiedlichen, vielfältigen Konfigurationen« (ebd.).

Diese Konfigurationen, in denen sich bspw. niederschlägt, ob kognitive Pluralisierung und institutionelle Entgrenzung auf Teilbereiche beschränkt bleiben, marginalisiert werden, kompromisshaft, unstrukturiert oder integriert verarbeitet werden (ebd.), werden unseres Erachtens wesentlich vom Zusammenspiel der Verarbeitung von Funktionskrisen und der Bewältigung von Legitimationskrisen geformt. Die Ausgestaltung des Meta-Wandels in den Phasen

18) Vgl. Zukunftsstiftung Landwirtschaft (2003). 
von Transformation und Übergang wird in anderen Worten vom Wechselspiel der intendierten Reform oder auch Revolution mit dem eher verborgen und reflexartig ablaufenden Kategorienwandel bestimmt.

Zweifellos sind mit den großen Lebensmittel- und Agrarkrisen einige wesentliche Überzeugungen der industriellen Agrarmoderne erodiert und haben auf der kognitiven Ebene einer Pluralität von sehr unterschiedlichen, nicht mehr zwangsläufig mit wissenschaftlichem Objektivitätsanspruch auftretenden Problemlösungsmodellen Raum gegeben, die ihrerseits in ein weites diskursives Feld von Vorstellungen über Sein und Sollen im Zusammenhang von Natur und Gesellschaft eingebunden sind. Andererseits operieren die zentralen Einrichtungen der modernen Agrarwissenschaft und -politik unter der generalisierten Erwartung, über besondere, rationale Kompetenzen im Umgang mit auftretenden Problemen und Ungewissheiten zu verfügen und letztere sukzessive zu verringern. Thomas Gieryn (1999) hat mit dem Konzept des »boundary work« all jene strategischen Anstrengungen aufgezeigt, mit denen Wissenschaftler die Grenze zwischen der scientific community und von außen kommenden Bedrohungen ihrer kognitiven Autorität aufrecht erhalten (bspw. gegenüber PseudoWissenschaft, Technik, Praxiswissen etc.). Im Rahmen seiner Untersuchungen wird deutlich, welchen erheblichen Anteil Abgrenzungs- und Legitimierungspraktiken als Darstellung exklusiver Kompetenz nicht nur in der Wissenskommunikation »nach außen « haben, sondern auch intern in der Bezugnahme auf die methodische Legitimierung von Einschätzungen und Handlungsvorschlägen. Darüber hinaus erwies sich das Konzept als fruchtbar, um auch die strategische Abgrenzungs- und Inszenierungsarbeit von politischen und administrativen Tätigkeiten zu untersuchen, bspw. in der Politikberatung oder im Zusammenspiel von Experten mit Akteuren der regulierenden Verwaltung (vgl. auch Guston 2001). In eine ähnliche Richtung gehen Überlegungen aus der Perspektive der mikropolitischen Organisations- und Professionalisierungssoziologie, die auf die machtpolitische Bedeutung der Kontrolle von für Interaktionspartner relevante Unsicherheitszonen in Organisationen verweisen (vgl. Küpper/Ortmann 1988). In einfachen Worten besteht die Einsicht darin, dass eine anerkannte und autorisierte Sprecherposition ihre Autorität nur solange wahren kann, wie sie zumindest vermeintlich über die Kompetenz verfügt, die Ungewissheit anderer in Bezug auf konkrete Lösungsmöglichkeiten reduzieren bzw. deren relevante Probleme an ihrer Stelle lösen zu können. Verschwimmen aber die Unterschiede oder scheinen die Wissensbestände austauschbar oder plural ineinander laufend, so gilt das gleiche für ihre Träger: ein Wissenschafts- oder Verwaltungsexperte, der in der Wahrnehmung der Politiker oder der Fachöffentlichkeit nur wiederholt, was diese »in etwa genauso gut« hätten sagen können, macht sich entbehrlich. Pflege und Darstellung der besonderen Kompetenz und Legitimität sind daher wesentlicher Bestandteil des Handelns in Organisationen jedweder Art.

Nun führte die politische Rede von der »notwendigen, tief greifenden Umorientierung « im Agrarbereich zu einer öffentlichen Delegitimierung und Deklassifizierung seiner Repräsentanten und ihrer Autorität. Zur Rettung des eigenen Kompetenzanspruchs wurde daher diese Rede fast zwangsläufig als ideologisch, undifferenziert und inkompetent zurückgewiesen und die Wende-Programmatik beinahe reflexhaft abgewehrt. Dieser schematischen Ableitung steht allerdings eine ebenso reflexhafte, eher schleichende Integration eines Teils des AgrarwendeProgramms gegenüber, die sich aus der nicht mehr hintergehbaren Pluralisierung ehemals unverfügbarer Gewissheiten ergibt. »Nachhaltigkeit« bspw. ist heute ein explizites Thema in jedem Forum zur Zukunft der Landwirtschaft, kann nicht länger als per se in der »guten fachlichen Praxis « gesichert übergangen werden. ${ }^{19}$ Um die Reichweite der Veränderungen richtig einzuschätzen, muss daher auf subtilere Weise in den betroffenen Institutionen geforscht wer-

19) So müssen die Vertreter der »konventionellen (sic!) Norm« nun defensiv argumentieren, dass sich Nachhaltigkeit und konventionelle Landwirtschaft nicht notwendig widersprechen (vgl. Sonnleitner 2001). 
den, reicht eine oberflächliche Diskussion des Policy-Outputs nicht aus. Dass ein Wandel des Bezugsrahmens erfolgte, ist bspw. weniger an der erstarkten Agraropposition abzulesen, die ihrerseits vielfach wenig reflexiv auf ihre Gewissheiten, ihre gegenkulturellen Netzwerke und ihr mitunter eindimensionales Sicherheitsversprechen unter umgekehrtem Vorzeichen pocht (vgl. a. Oosterveer 2002), als an dem simplen Tatbestand, dass heute für Agrarfragen grundsätzlich eine Öffentlichkeit besteht, Legitimationsnotwendigkeiten herrschen, wo noch vor wenigen Jahren agrarökonomische Gewissheiten jede Diskussion im Keim erstickten.

\section{Nebenfolgendynamik und institutioneller Meta-Wandel}

Das gezielte politische Umsteuern mit der Berufung von Renate Künast nicht zur neuen Landwirtschaftsministerin, sondern zur Verbraucherschutzministerin ging mit dem Versuch einher, das Feld neu zu fassen und einen prinzipiellen Richtungswechsel zu etablieren: Mit dem politischen Leitbild des vorsorgenden Verbraucherschutzes drehte sich der bisherige, in den institutionellen Formen festgeschriebene Blick herum, statt landwirtschaftlicher Produktion und verbesserter Wettbewerbsfähigkeit stand nun Verbraucherschutz im Zentrum. ${ }^{20}$ Dieses Projekt verabschiedet - wie in der Theorie der reflexiven Modernisierung angekündigt - endgültig die Orientierung an Reichtumsverteilung und stellt stattdessen auf Abwehr und Vermeidung von Risiken und Gefährdungen um. Eine solche fundamentale Perspektivenverschiebung, die zentral das Unerwartete und die Nebenfolge erwartet, ist ihrerseits mit weit mehr »Nebenfolgen« verbunden, als dies der einfache Zielsatz und der bloße Namenswechsel des Ministeriums andeuten. Auch wenn die Agrarwende-Rhetorik den Anschein erweckte, dass nun einfach ein Klientelkreis durch einen anderen ersetzt wurde, gerieten tradierte Gewissheiten damit in die Mühlen der Pluralisierung von Einflussstrukturen und Problemsichten. ${ }^{21}$ Heute zeigt sich, dass

20) Man wollte nicht länger (nur) ein Ministerium der Agrarier sein - das bis dahin übliche MinisteriumKürzel BMEL wurde von Spöttern gerne mit »Bundesministerium zur Ernährung der Landwirtschaft« übersetzt -, sondern ein Ministerium der Verbraucher, das »dem Schutz vor Gesundheitsgefährdung Vorrang vor anderweitigen, insbesondere wirtschaftlichen Interessen « (BMVEL 2005) einräumt.

21) Von Seiten der alten Agrareliten wurde nicht zu unrecht darauf hingewiesen $»$ dass auch die neue Agrarpolitik Klientelpolitik ist, nur hat die Klientel gewechselt« (von Alvensleben 2003: 1). Dies zeigt, dass der Weg nicht zur Behauptung einer »unklientelistischen«, rein am Bürgerinteresse orientierten Politik zurückführen kann. Vielmehr ginge es darum, die unterschiedlichsten Interessen als solche zu kennzeichnen, einzubeziehen und zueinander ins Verhältnis zu setzen. Ähnliches gilt für das Verhältnis zwischen Wissenschaft und Politik. Als Renate Künast im Juni 2001 die Umstrukturierung des wissenschaftlichen Beirates des Ministeriums ankündigte, äußerte sie die Vorstellung, dass zukünftig das Ministerium dessen Mitglieder bestimmten solle. Die Folge war der Rücktritt des kompletten Beirates, der darin eine unbotmäßige Einmischung der Politik in die Domäne des wissenschaftlichen Sachverstandes sah (vgl. FAZ vom 23.1.2001.) Tatsächlich hat die Kritik an der einseitigen Ausrichtung des bisherigen Beirates es der Ministerin schwer ermöglicht, nun ihrerseits Wissenschaftler nach politischen Kriterien - auf dem grünen Ticket - auszuwählen. Der zwangsläufige Ausweg war schließlich auch hier Pluralisierung und Dialogorientierung: zum einen blieb es dem neuen Beirat vorbehalten, seine eigenen Mitglieder zu bestimmen, zum anderen gab es jedoch eine deutliche fachliche Verbreiterung. Setzte sich der bisherige Beirat vornehmlich aus Agrarökonomen und Tiermedizinern zusammen, so gehören ihm nun auch LandschaftsökologInnen und WissenschaftlerInnen aus dem Bereich der Ländlichen Entwicklung und der Raumplanung sowie VertreterInnen der Wissenschaft zu Ökologischem Landbau an. Gleichzeitig wurde ihm ein Beirat für Verbraucherschutzfragen gegenüber gestellt: das Feld der wissenschaftlichen Politikberatung im Agrarbereich, früher fest in der Hand der Agrarökonomen, wird somit von einer Vielzahl unterschiedlicher Stimmen und Sichtweisen geprägt, die - wollen sie Relevanz entwickeln - sich dialogisch aufeinander beziehen und abstimmen müssen. Welche Folgen dies für die Bewältigung der Aufgaben der Beiräte haben wird, bleibt abzuwarten. Ein Mitglied des neuen agrarpolitischen Beirates hat im Interview darauf hingewiesen, dass der Verhandlungsbedarf innerhalb des Gremiums massiv zugenommen habe und deutliche Verständigungsschwierigkeiten zwischen den unterschiedlichen Gruppierungen zu beobachten seien. Die Effizienz der Politikberatung habe damit abgenommen. Gleichzeitig ließen sich jedoch Konflikte, die früher erst nach Veröffentlichung der wissenschaftlichen Stellungnahmen entstanden, nun innerhalb des Gremiums bearbeiten. 
sich der Agrarbereich unwiderruflich für neue Sprecher und Positionen geöffnet hat, seine Politisierung durch einen deutlich gewachsenen Kreis legitimierter Akteure betrieben wird und eine Rückkehr zu Ruhe und Eindeutigkeit vor der Krise nicht mehr erwartbar ist.

In Krisenzeiten werden gerne alle betroffenen Akteure an einen Tisch gerufen. So setzte auch Renate Künast für die Entwicklung und Umsetzung ihrer zukünftigen Politik auf ein »magisches Sechseck« aus Verbrauchern, Landwirten, Futtermittelindustrie, Lebensmittelindustrie, Einzelhandel und Politik. Alle gemeinsam, so das Ziel, sollten entlang der - nun gläsernen - Produktionskette an einem Strang ziehen, um Lebensmittelsicherheit und Verbrauchervertrauen zurück zu gewinnen. So eingängig die Idee ist, so klar ist auch, dass die beteiligten Stakeholder sehr unterschiedliche, sich teilweise widersprechende Interessen haben. Erfahrungen mit partizipativen Verfahren und runden Tischen haben gezeigt, das diese die Frage der Integration von Sichtweisen, Interessen und Orientierungen nicht unbedingt lösen, sondern oft erst verdeutlichen und manifest auf die Tagesordnung setzen (vgl. Wagner 2003: 138f). So wirft etwa die Einführung von Qualitätssicherheitsmanagementsystemen, die die ganze Produktionskette betreffen, eine ganze Reihe von Konflikten unterschiedlicher Produktionslogiken und Arbeitsorganisationen auf (vgl. Schmidt/Jasper 2001: 148ff.). Die auf den arbeitsteilig organisierten, nachgelagerten Bereich hin zugeschnittenen Systeme der Dokumentation und Standardisierung stellen für viele Landwirte eine erhebliche Belastung dar, die kaum in den Arbeitsalltag zu integrieren ist. Zudem ist das Verständnis von Qualität aus der Logik der unterschiedlichen Akteursgruppen entlang der Kette ein je anderes und oft widersprüchliches, für die Lebensmittelindustrie etwa »Keimfreiheit «, für den Verbraucher aber »Naturnähe«. Wie in ähnlichen Fällen fürchten die Lebensmitteltechniker die Natur, die Verbraucher aber die Lebensmitteltechnik. Nicht nur in dieser Frage lässt sich die Entwertung alter Strukturen und Deutungsmuster nicht einfach durch die Erzeugung neuer kompensieren, sondern geht in weitverzweigten Nebenfolgenreflexionen mit konfliktreichen Neuaushandlungen einher, die oft deutlich weniger eindeutige Strukturen hervorbringen. In der reflexiven Moderne macht Entgrenzung Entscheidungen notwendig, die sich immer weniger auf Selbstverständlichkeiten zurückziehen können.

Diese folgenreiche Einsicht mussten auch die »Wendegewinnler« machen: Das von der Ministerin formulierte Ziel, den Anteil des ökologischen Landbaus bis 2010 auf 20 Prozent anzuheben, führte dazu, dass eine alternative Gegen-Konzeption gegenüber den etablierten Strukturen nicht nur Anerkennung, sondern auch politisches Gewicht gewann. Auf diese Weise sind seit 2001 zumindest zwei »Wahrheiten« im Spiel, die zuvor als einander ausschließende Gewissheiten in einem ungleichen Wettbewerb standen. Mit der politischen Adelung des ökologischen Landbaus als Alternative, wurde zumindest implizit deutlich, dass Produktivitätssteigerung und internationaler Wettbewerb keineswegs naturwüchsiges oder praktisches Resultat der Moderne oder ihrer wissenschaftlichen Einsichten sind, sondern das Ergebnis bestimmter (agrarökonomischer) Perspektiven und konkreter politischer Konstellationen. Mit dem Motto »Agrarwende« wurden die Spielregeln innerhalb der Agrar-Arena tief greifend verändert. Selbst der scheinbare Gewinner der intendierten Wende, der ökologische Landbau, geriet im Zuge seiner Anerkennung und Verbreitung seinerseits unter Veränderungs- und Legitimationsdruck. Auch hier kommen Grenzziehungen und Selbstverständlichkeiten in Bewegung, beklagen die Pioniere des ökologischen Landbaus eine Aufweichung der Standards und den Verlust der der Bewegung zugrunde liegenden post-materialistischen Wertorientierung. Die Hoffnung, mit »bio« Geld zu machen, lässt andere, stärker gewinnorientierte Akteure in das Feld eindringen. Die unterschiedlichen Anbauverbände vertreten heute mehr als einen Problemlösungsanspruch, stehen vor der Herausforderung, sich gegenseitig voneinander abzugrenzen, wollen sie ihr Selbstverständnis, ihre Existenzberechtigung und vor allem ihr Marktsegment verteidigen. In der Konkurrenz wächst auch der Begründungsaufwand für die selbst gewählten Kriterien, etwa des Ausschlusses von Gentechnik in der ökologischen Landwirtschaft. Pluralisierung und Verlust fragloser Eindeutigkeit bleibt also keineswegs auf die bisher domi- 
nanten Strukturen beschränkt, sondern zersetzt in der gleichen Bewegung auch das Selbstverständnis der »Nischeneinigkeit«. So ist es nach dem Ende des einen, hegemonialen Entwicklungsparadigmas in der Landwirtschaft zu einem »bereichsspezifischen Pluralismus« (Beck/ Lau in diesem Heft: 20) gekommen, im Rahmen dessen die ehemals standardisierten Grenzdefinitionen für das gesamte institutionelle Feld durch eine Vielzahl nun konkurrierender Definitionen ersetzt wurden, die sich nur mehr auf stets neu von einander abzugrenzende Teilbereiche beziehen und mit unterschiedlichen Motiven »Grenzpolitik« betreiben.

\section{Unsicherheit als ständiger Gast}

Da die angestrebte Neuausrichtung der Agrarpolitik als Reaktion auf eine heftige Krise erfolgte, war mit ihr nicht zuletzt das Versprechen verbunden, die durch die Skandale als Illusion erscheinende Sicherheit im Lebensmittelbereich nun durch neue Maßnahmen wieder zu gewinnen. Das Leitbild des vorsorgenden Verbraucherschutzes sollte dafür Sorge tragen, dass Lebensmittelsicherheit über Wirtschaftsinteressen gestellt wird (vgl. Fußnote 20). Diese Rhetorik verdeckt, dass mit dem Prinzip der Vorsorge auch ein völlig anderes Verständnis im Umgang mit Risiken verbunden ist (vgl. auch Holzer/May in diesem Heft), das gerade nicht auf die Vermeidung von Unsicherheit, sondern auf den bewussten Umgang damit abzielt. Im Kern des Vorsorgeprinzips steht die »Regel des öffentlichen politischen Handelns in Situationen, die eine mögliche [...] Gefährdung der Umwelt oder der Gesundheit darstellen« (Gee/Guedes/Vaz 2004: 14. Herv. durch C.K/J.W.). Es zielt also auf politisches Handeln unter Unsicherheit ab, das sich auf keinen (wissenschaftlichen) Beweis berufen kann, sondern nur auf die Möglichkeit einer Gefahr. Im Gegensatz zur bisherigen Logik der mehr oder weniger objektiven Risikoabschätzung aufgrund von Fakten muss sich ein vorsorgender Gesundheitsschutz viel stärker auf das Abwägen von möglichen Risiken und potentiellen Gefahren sowie von bewusster Vermeidung oder Hinnahme - nicht nach, sondern vor dem Entscheiden - beziehen. Vor diesem Problem werden auch die neu geschaffenen Institutionen im Bereich Lebensmittelsicherheit stehen.

Es ließen sich weitere Konkretisierungen für das komplexe Ineinandergreifen von intendierter Wende und schleichendem Wandel, von Reform und Transformation, von intendierter und schleichender Anerkennung von Ungewissheit und Ambivalenz sowie von intendierter Öffnung für Alternativen und schleichender Pluralisierung bestehender Eindeutigkeiten und Grenzziehungen (auch der Alternativen) anführen, bspw. durch die Öffnung für eine »transdisziplinäre Agrarwissenschaft«. Wir möchten hier aber die Konsequenzen unserer Diskussion für die eingangs gestellte Frage resümieren, als wie tief greifend der Wandel durch die Agrarwende zu betrachten ist und in wie weit damit von »reflexiver Modernisierung « im Agrarbereich zu sprechen ist.

\section{Fazit}

Zwischen Krise und Normalbetrieb liegen je nach Themenkonjunktur mehr oder weniger flüchtige Episoden, in denen wesentliche Elemente des institutionellen Status quo delegitimiert und die Kompetenzen der institutionalisierten Sicherheitsgaranten herausgefordert sind. Die Krisen-Forscher Paul 't Hart und Arjen J. Boin (2001) betrachten diese restorativ oder innovativ nutzbaren Phasen als »the long shadow of post-crisis politics«. Ganz im Sinne des altgriechischen Verständnisses von (produktiven) Krisen eröffnen solche Episoden oder Übergänge oftmals politische, soziale und moralische Möglichkeiten, »windows onto previously unsuspected weaknesses in the social matrix « (vgl. Jasanoff 1994: 2). Inwieweit die zumindest kurzfristig generierten Brüche der sozialen Ordnung für einen der reflexiven Moderne angemessenen Wandel genutzt werden können, hängt von den in diesen schwierigen Zeiten generierten und im Laufe des wiedergekehrten Normalbetriebs verstetigten institutionellen 
Fähigkeiten ab, folgensensibel, reflexiv und dialogorientiert mit den unhintergehbaren Ungewissheiten und den kategorialen Ambivalenzen umzugehen (vgl. dazu Wagner/Kropp 2005).

Wie tief greifend der soziale und politische Wandel im Gefolge von Krisen ist, wird aus der in diesem Artikel verfolgten Perspektive nicht alleine von den üblicherweise diskutierten Gelegenheitsstrukturen bestimmt, nicht von der Ehrlichkeit der Absichten, den bestehenden Verhandlungssystemen oder der Tiefe bzw. Härte der ergriffenen Maßnahmen. Vielmehr entscheidet das Wechselspiel von intendierter Wende und schleichend verlaufendem Meta-Wandel über »Integration und Ableitung «. Wie anhand der großen Agrarskandale dargestellt, vollziehen sich Prozesse der Pluralisierung und Entgrenzung so oder so, ungewollt, unentschieden, im Rahmen der radikalisierten, modernen Eigendynamiken, der nicht-intendierten Nebenfolgen einfacher Modernisierung, der damit losgetretenen Nebenfolgenkaskaden und -dynamiken sowie durch »Prozesse der Reflexion auf die Grundlagen der Ersten Moderne« (Beck/Lau in diesem Heft: 13). Im Krisenmanagement von BSE und MKS wurden diese Prozesse aufgegriffen, politisch übersetzt und gerichtet in ein überfordertes institutionelles Feld zurückgegeben. Nun erschienen sie aber nicht mehr als blinde Kraft einer wie auch immer wahrgenommenen »reflexiven Modernisierung «, sondern als politischer Wille. Als solcher gerieten sie unter dem Etikett »Agrarwende « selbst in den Sog der Pluralisierung, wurden der propagierten Eindeutigkeit und Zwangsläufigkeit entkleidet und in noch andauernde Auseinandersetzungen gezogen, deren Ende nicht absehbar ist. Weil darüber hinaus Institutionen und Organisationen gerade im Moment der Krise unter massivem Entscheidungsdruck stehen, an den sich Verantwortungsund Haftungsfragen koppeln, erscheint es doppelt attraktiv, zur Eindeutigkeit zurückzukehren und jeden Wandel auszubremsen.

»Entweder es wird die Herrschaft der alten Grenzen zu erneuern versucht; es sei dezisionistisch, sei es fundamentalistisch; oder aber es beginnt ein Lernprozess der Institutionen, in dessen Verlauf reflexive Verfahren im Umgang mit Unsicherheit, Ungewissheit und Ambivalenz entwickelt und umgesetzt werden. Was sich durchsetzt, ist gänzlich offen (hoffentlich!), entscheidet sich jedoch nicht zuletzt daran, inwieweit die Sowohl-als-auch-Logik als eine Erweiterung und gerade nicht als ein Verlust von Handlungsoptionen entdeckt und begriffen wird.« (Beck/Lau in diesem Heft).

Wir vermuten, dass die proklamierte »Agrarwende « dort am erfolgreichsten ist, wo sie als schleichender, aushöhlender Wandel am wenigsten sichtbar vollzogen wird. Institutionen müssen weiterhin Zuordnungen und Grenzentscheidungen treffen, müssen ihren zeitlichräumlich-sachlichen Zuständigkeitsbereich abgrenzen, um Legitimationsschwierigkeiten und vernichtende Verantwortungszumutungen abzuwehren. Wo dies aufgrund zersetzter Leitlinien, pluralisierter Grenzbestimmungen und unscharfer Handlungsorientierungen nicht mehr möglich ist, suchen sie fallbezogen nach Alternativen, nach neuen Bündnissen, neuen Rationalitäten und Selektivitäten und setzen sich nolens volens mit der vorgefundenen Hegemonie des industriellen Entwicklungsparadigmas auseinander. Zentral haben sie dabei die Frage nach Begründungs- und Legitimationsformen zu beantworten - diese interne Funktionsnotwendigkeit könnte schließlich auch das politisch formulierte Programm der »Agrarwende« attraktiv machen.

\section{Literatur}

Beck, U. (1986): Risikogesellschaft. Auf dem Weg in eine andere Moderne. Frankfurt/Main.

Beck, U. (1988): Gegengifte - Die organisierte Unverantwortlichkeit. Frankfurt/Main.

Beck, U. (1993): Die Erfindung des Politischen. Frankfurt/Main.

Beck, U./Bonß, W. (2001): Die Modernisierung der Moderne. Frankfurt/Main.

Beck, U./Bonß, W./Lau, Chr. (Hrsg.) (2001): Theorie reflexiver Modernisierung - Fragestellungen, Hypothesen, Forschungsprogramme, in: U. Beck /W. Bonß (Hrsg.): Die Modernisierung der Moderne. Frankfurt/Main, S. 11-59.

Beck, U./Grande, E. (2004): Das kosmopolitische Europa. Frankfurt/Main. 
Beck, U./Lau, Chr. (Hrsg.) (2004): Entgrenzung und Entscheidung. Was ist neu an der Theorie reflexiver Modernisierung? Frankfurt/M.

BMBF, Bundesministerium für Bildung und Forschung (2002): Chance für Deutschland und Europa. Das 6. Forschungsrahmenprogramm. Bonn.

BMVEL, Bundesministerium für Verbraucherschutz, Ernährung und Landwirtschaft (2005): Die Verbraucherpolitik der Bundesregierung. eingesehen am 25.01.2005: http://www.verbraucherschutzministerium.de.

Böschen, S./Dressel, K./Schneider, M./Viehöver W. (2002): Pro und Kontra der Trennung von Risikobewertung und Risikomanagement - Diskussionsstand in Deutschland und Europa, TAB-Gutachten, München.

Böschen, S./Viehöver, W./Zinn, J. (2002): Rinderwahnsinn: Können Gesellschaften aus Krisen lernen? in: Berliner Journal für Soziologie 13, S. 35-58.

Boin, R.A./t'Hart, P. (2000): Institutional Crisis and Reforms in Policy Sectors. In: Wagenaar, H. (Hg.): Government Institutions: Effects, Changes and Normative Foundations. Dordrecht. S. 9-31.

Bundesamt für Naturschutz, (2002): Denkschrift. »Forschung für eine naturgerechte Landwirtschaft.« Bonn.

Carson, R. (1962): Silent Spring. Boston.

Dewey, J. (2001): Die Öffentlichkeit und Ihre Probleme, Berlin.

Douglas, M. (1991): Wie Institutionen denken. Frankfurt/Main.

Dressel, K. (2001): The Surveillance of BSE and CJD in Germany. Studie im Auftrag der WHO, München.

Eder, K. (1998): Gibt es Regenmacher? Vom Nutzen des Konstruktivismus in der soziologischen Analyse der Natur. In: K.-W. Brand (Hrsg.): Soziologie und Natur. Theoretische Perspektiven. Opladen, S.97-115.

Feindt, P./Ratschow, Chr. (2003): »Agrarwende«: Programm, Maßnahmen und institutionelle Rahmenbedingungen. BIOGUM Forschungsbericht Nr.7, Hamburg.

Gee, D./Guedes Vaz, S. (2004): Späte Lehren aus frühen Warnungen. Das Vorsorgeprinzip 1896-2000, Berlin.

Gerlach, S./Kropp, C./Spiller, A./Ulmer, H. (2005): Die Agrarwende - Neustrukturierung eines Politikfeldes. Arbeitspapier 5 des BMBF-Forschungsprojekts »Von der Agrarwende zur Konsumwende?«. Göttingen/München. Manuskript.

Giddens, A. (1988): Die Konstitution der Gesellschaft. Grundzüge einer Theorie der Strukturierung. Frankfurt/Main.

Giddens, A. (1991): Modernity and Self-Identity. Self and Society in the Late Modern Age. Cambridge.

Gieryn, T. (1999): Cultural boundaries of science: credibility on the line, Chicago.

Göhler, G. (1994): Die Eigenart der Institutionen. Zum Profil politischer Institutionentheorie. Baden-Baden.

Guston, D. (2001): Boundary Organizations in Environmental Policy and Science: An Introduction, in: Science, Technology and Human Values 26, S. 399-408.

Hajer, M. (2004): Food for Thought: A Comparative Study of Administrative Innovations in Food Safety Regulation in Western Europe after the BSE Crisis. Research Proposal.

Heinze, R.G./Voelzkow, H. (1994): Der Deutsche Bauernverband im Spannungsfeld von Ökonomie und Ökologie, in: in: Schriften der Gesellschaft für Wirtschafts- und Sozialwissenschaften des Landbaus e.V. 30, S.163-172.

Henrichsmeyer, W./Witzke, H.P. (1991): Agrarpolitik, Bd. 1, Stuttgart.

Hinchliffe, S. (2001): Indeterminacy in decisions: science, policy and politics in the BSE crisis. In: Transactions of the Institute of British Geographers 26, S. 182-204.

Hohn, H.-W./Schimank, U. (1990): Konflikte und Gleichgewichte im Forschungssystem. Akteurkonstellationen und Entwicklungspfade in der staatlich finanzierten außeruniversitären Forschung, Frankfurt/ Main. 
Initiativkreis Agrar- und Ernährungsforschung (2002): Bonner Erklärung. Bonn.

Isermeyer, F. (2001): Kurswechsel in der Agrarpolitik - Umorientierung in der Agrarforschung? FALArbeitsbericht. Braunschweig.

Isermeyer, F. (2003): Für eine leistungsfähige Agrarforschung in Deutschland. FAL-Arbeitsbericht, Braunschweig.

Jasanoff, Sh. (1994): Introduction: Learning from Disaster. In: dies. (Ed.): Learning form Disasters: Risk Management After Bhopal. Philadelphia. S. 1-21.

Kleinschmidt, D./Feindt, P. (2004): Verursacher, Opfer und Helfer. BSE und Agrarpolitik in deutschen Zeitungen, in: Forschungsjournal Neue Soziale Bewegungen 17, S. 93-97.

Kötter, H. (1997): Agrarwissenschaften in der Postmoderne: Versuch einer Orientierung unter Einbeziehung wirtschafts- und sozialethischer Aspekte. Forschungsstelle für Internationale Agrar- und Wirtschaftsentwicklung e.V. Paper 56, Heidelberg.

Kropp, C. (2002): »Natur«. Soziologische Konzepte, politische Konsequenzen. Opladen.

Küpper, W./Ortmann, G. (1988): Mikropolitik. Rationalität, Macht und Spiele in Organisationen. Opladen.

Kuhlmann, F. (1996): Aufgaben und Leistungen der Agrarforschung für Landwirtschaft und Gesellschaft, in: Dachverband der Agrarforschung (Hrsg.): Standortbestimmung und Perspektiven der agrarwissenschaftlichen Forschung, Agrarspectrum 25, Frankfurt/Main, S. 42-54.

Latour, B. (1990): The Force and the Reason for Experiment, in: H.E. Le Grand (Hrsg.): Experimental Inquiries. Historical, Philosophical and Social Studies of Experimentation in Science, Dodrecht, S.49-80.

Latour, B. (1995): Wir sind nie modern gewesen: Versuch einer symmetrischen Anthropologie. Berlin.

Latour, B. (2001): Das Parlament der Dinge. Für eine politische Ökologie. Frankfurt/Main.

Latour, B. (2003): Is Re-modernization Occurring - And If So, How to Prove It? A Commentary on Ulrich Beck, in: Theory, Culture \& Society 20, S. 35-48.

Latour B. (2004): Ein Experiment von und mit uns allen, in: Gamm, A./Hetzel, A./Lilienthal, M. (Hg.): Die Gesellschaft im 21. Jahrhundert. Perspektiven auf Arbeit, Leben, Politik. Frankfurt/Main.

Luhmann, N. (1991): Soziologie des Risikos. Berlin.

Mante, J. (2005): Wandel im Selbstverständnis der Agrarforschung?, in: Kropp C./Schiller, F./Wagner, J. (2005): Neue Formen des Dialoges von Wissenschaft und Politik im Agrarbereich: auf dem Weg zu reflexiver und folgensensibler Schnittstellenkommunikation? Berlin. In Vorbereitung.

Mayntz, R. (1999): Wissenschaft, Politik und die politischen Folgen kognitiver Unsicherheit, in: J. Gerhards/R. Hitzler (Hrsg.): Eigenwilligkeit und Rationalität sozialer Prozesse, Opladen, S. 30-45.

Meadows, D. (1972): The Limits to Growth. New York.

Merchant, C. (1987): Der Tod der Natur. Ökologie, Frauen und neuzeitliche Naturwissenschaft. München.

Mol, A. P.J./Bulkeley, H. (2002): Food Risks and the Environment: Changing Perspectives in a Changing Social Order. In: Journal of Environmental Policy \& Planning 4, S. 185-195.

Niggli, U. (1999): Ganzheitliche Ansätze für die Forschung - ein Überblick, in: Ökologie \& Landbau 27, S. 38-39.

Ober, S. (2004): Agrarforschung in Deutschland. Eine Studie im Auftrag der Zukunftsstiftung Landwirtschaft. Berlin.

Offe, C. (1989): Bindung, Fessel, Bremse. Die Unübersichtlichkeit von Selbstbeschränkungsformeln. In: Honneth, A. (Hrsg.): Zwischenbetrachtungen. Im Prozess der Aufklärung. Frankfurt am Main: Suhrkamp, S. 739-774.

Oosterveer, P. (2002): Reinventing Risk Politics: Reflexive Modernity and the European BSE Crisis. In: Journal of Environmental Policy \& Planning 4, S. 215-222.

Ortmann, G. (2004): Als ob. Fiktionen und Organisationen. Opladen.

Pior, A. (2000): Agrarforschung im Wandel. Eine Analyse im internationalen Vergleich. Agrarspectrum. Schriftreihe des Dachverbandes der Agrarforschung. Band 32. Frankfurt/Main. 
Renn, O./Dreyer, M./Klinke, A. (2002): Systemic Risks. Report to the Steering Group of the OECD Futures Project on Emerging Systemic Risks, Stuttgart.

Rieger, E (1995): Bauernopfer. Das Elend der europäischen Agrarpolitik. Frankfurt/Main.

Rifkin, J. (2001): Das Imperium der Rinder, Frankfurt/Main.

Schmidt, G./Jasper, U. (2001): Agrarwende oder die Zukunft unserer Ernährung, München.

Scott, J.C. (1999): Seeing Like a State: How Certain Schemes to Improve the Human Condition Have Failed. New Haven, CT.

Sonnleitner, G. (2001): Neue Agrarpolitik - Ökologischer Landbau, in: U. Simonis (Hrsg.): Jahrbuch für Ökologie 2002, München, S.22-28.

Tacke, V. (2001): BSE as an organizational construction: a case study on the globalization of risk. In: British Journal of Sociology. Vol. No. 52, S. 293-312.

Thaer, A. (1809): Begründung der Lehre und des Gewerbes: Oekonomie oder die Lehre von den landwirthschaftlichen Verhältnissen. Grundsätze der rationellen Landwirthschaft Bd.1, Berlin.

't Hart, P./Boin, A. R. (2001): Between Crisis and Normalcy: The long shadow of post-crisis politics. In: Rosenthal, U. et al. (Eds.): Managing Crisis - Threats, Dilemmas, Opportunities. Springfield. S. 28-46.

Thielen, H. (1991): Agraropposition in Deutschland - eine neue politische Kraft? in: Utopie Kreativ 15. S. 46-52.

Viehöver, W. (2000): La Mucca Pazza. Rinderwahnsinn als Nebenfolge moderner Viehzucht und ihre Konsequenzen für die Auflösung der Differenz Natur/Gesellschaft in der Moderne. Arbeitspapier 3 zum Projekt »Vergesellschaftung der Natur und Naturalisierung der Gesellschaft« im Rahmen des SFB $»$ Reflexive Modernisierung «, Augsburg.

Viehöver, W./Gugutzer, R./Keller, R./Lau, Chr. (2004): Vergesellschaftung der Natur - Naturalisierung der Gesellschaft. In: Beck, U./Lau, Chr. (Hrsg.): Entgrenzung und Entscheidung. Edition Zweite Moderne. Frankfurt. S. 65-94.

von Alvensleben, R. (2003): Kritik der neuen Agrarpolitik, Diskussionsbeitrag zum Verbraucherpolitischen Forum der Verbraucherzentrale Bundesverband e.V. auf der Internationalen Grünen Woche am 23.1.2003 in Berlin., in: Agrar-Europe 4/03.

von Wedel, H. (2001): Organisation des gesundheitlichen Verbraucherschutzes (Schwerpunkt Lebensmittel). Stuttgart.

Wagner, J. (2003): Risikokonstruktionen in partizipativen Verfahren der Technikfolgenabschätzung, Magisterarbeit, Berlin.

Wagner, J./Kropp, C (2005): Dimensionen einer dialogisch-reflexiven Wissenserzeugung und -kommunikation im Agrarbereich. in: Kropp, C./Schiller, F./Wagner, J. (2005): Neue Formen des Dialogs von Wissenschaft und Politik im Agrarbereich: auf dem Weg zu reflexiver und folgensensibler Schnittstellenkommunikation? Berlin. In Vorbereitung.

Ziche, J./Kromka, F. (1982): Gesellschaftliche Veränderungen - wird die Landwirtschaft zum Sündenbock? in: Schriften der Gesellschaft für Wirtschafts- und Sozialwissenschaften des Landbaus e.V. 19, S.103-124.

Zukunftsstiftung Landwirtschaft (2003): Die Farbe der Forschung. Symposium, Berlin.

Dr. Cordula Kropp,

Münchner Projektgruppe für Sozialforschung, Dachauerstr. 189, 80637 München, cordula.kropp@sozialforschung.org

Jost Wagner,

Münchner Projektgruppe für Sozialforschung (MPS),

Dachauer Str. 189, 80637 München. jost.wagner@sozialforschung.org 